\title{
Lo studio internazionale multicentrico dell'Organizzazione Mondiale della Sanità sui disturbi psichici nella medicina generale: risultati relativi all'area di Verona ${ }^{1}$
}

\author{
The World Health Organization international multicentre study on psychological \\ problems in general health care settings: results in the area of Verona
}

\author{
MARCO PICCINELLI ${ }^{1}$, STEFANO PINI ${ }^{1}$, CESARIO BELLANTUONO', \\ PAOLA BONIZZATO ${ }^{1}$, ELISABETTA PALTRINIERI ${ }^{1}$, T. BEDIRHAN ÜSTÜN ${ }^{2}$, \\ NORMAN SARTORIUS ${ }^{2}$, MICHELE TANSELLA ${ }^{1}$ \\ ${ }^{1}$ Servizio di Psicologia Medica, Istituto di Psichiatria, Università di Verona, Verona \\ ${ }^{2}$ Division of Mental Health, World Health Organization, Geneva
}

\begin{abstract}
Riassunto. Obiettivi - Presentare i risultati emersi dalla valutazione trasversale di un campione di soggetti selezionato negli ambulatori di medicina generale dell'area di Verona nel corso dello studio internazionale multicentrico dell'Organizzazione Mondiale della Sanità sui disturbi psichici nella medicina generale. Metodo - Tra coloro che si erano rivolti consecutivamente agli ambulatori di 16 medici di base nel periodo aprile 1991/febbraio 1992, è stato selezionato un campione randomizzato di soggetti, stratificato sulla base del punteggio riportato al GHQ-12, da sottoporre ad un'approfondita valutazione dello stato psicopatologico, dello stato di salute fisica e dei livelli di disabilità in campo occupazionale e nelle altre attività quotidiane. Tutti i pazienti che alla valutazione iniziale presentavano sintomi psicopatologici ed un campione di soggetti pari al $20 \%$ di coloro che non presentavano sintomi psicopatologici sono stati rivalutati a distanza di 3 mesi e poi ancora di un anno (dati non presentati in questa sede). Risultati - In totale, dei 1.656 soggetti contattati dai ricercatori negli ambulatori di medicina generale, 1.625 soddisfacevano i requisiti richiesti per essere inclusi nello studio. La procedura di screening è stata completata da 1.558 soggetti e la valutazione del secondo stadio da 250 . Disturbi psichici secondo i criteri dell'ICD-10 erano presenti nel $12.4 \%$ dei soggetti che avevano contattato consecutivamente gli ambulatori di medicina generale e, di questi, un terzo circa (4.5\% dei soggetti sottoposti inizialmente a screening) soddisfaceva i criteri diagnostici per la presenza contemporanea di due o più disturbi psichici diversi. Le diagnosi di Episodio Depressivo Attuale (4.7\%) e quella di Sindrome Ansiosa Generalizzata (3.7\%) sono risultate le più frequenti. Un ulteriore $11.2 \%$ dei soggetti selezionati presentava disturbi psichici 'sotto la soglia' (così definiti sulla base della presenza di sintomi in almeno due aree sintomatologiche distinte tra quelle previste dalle singole categorie diagnostiche dell' ICD-10, senza che venissero però soddisfatti tutti i criteri diagnostici per la presenza del disturbo in esame). I disturbi psichici sono risultati più frequenti tra le donne e nella classe di età compresa tra 25 e 44 anni. Solamente il $20.6 \%$ dei soggetti con
\end{abstract}

Indirizzo per la corrispondenza: Dr. M. Piccinelli, Servizio di Psicologia Medica, Istituto di Psichiatria, Ospedale Policlinico, 37134 Verona.

Fax (+39) 45-585.871.

(1) Questo lavoro si basa sui dati raccolti nel corso del Progetto Psychological Problems in General Health Care Settings, che è stato coordinato dall'Organizzazione Mondiale della Sanità e finanziato dalla stessa e dai Centri partecipanti. I ricercatori responsabili del Progetto sono stati: Dr. N. Sartorius e Dr. T.B. Üstün, Division of Mental Health, WHO, Ginevra (Svizzera), Dr. O. Özturk e Dr. M. Rezaki, Ankara (Turchia), Dr. C. Stefanis e Dr. V. Mavreas, Atene (Grecia), Dr. S.M. Channabasavanna e Dr. T.G. Sriram, Bangalore (India), Dr. H. Helmchen e Dr. M. Linden, Berlino (Germania), Dr. W. van den Brink e Dr. B. Tie- mens, Groningen (Olanda), Dr. M. Olatawura e Dr. O. Gureje, Ibadan (Nigeria), Dr. O. Benkert e Dr. W. Maier, Mainz (Germania), Dr. D. Goldberg e Dr. R. Gater, Manchester (Gran Bretagana), Dr. Y. Nakane e Dr. S. Michitsuji, Nagasaki (Giappone), Dr. Y. Lecrubier e Dr. P. Boyer, Parigi (Francia), Dr. J.A. Costa e Silva e Dr. L. Villano, Rio de Janeiro (Brasile), Dr. R. Florenzano e Dr. J. Acuna, Santiago del Cile (Cile), Dr. M. von Korff e Dr. G. Simon, Seattle (USA), Dr. Yan He-Qin e Dr. Xaio Shi Fu, Shangai (Cina), Dr. M. Tansella e Dr. C. Bellantuono, Verona (Italia). Un elenco completo dei ricercatori e del personale che ha fornito il proprio contributo durante lo svolgimento del Progetto è riportato nella monografia Mental Illness in Primary Care. An International Study (ed. N. Sartorius and T.B. Üstün), attualmente in stampa. 
disturbi psichici si erano rivolti al medico per i loro sintomi psicopatologici, il 5.7\% lamentavano sintomi di possibile origine psicopatologica, mentre nel $70 \%$ circa dei casi il disturbo psichico è risultato mascherato dietro la presentazione di sintomi somatici, di dolori in varie parti del corpo oppure di disturbi organici cronici. Il $62.0 \%$ dei soggetti con disturbi psichici hanno valutato la propria salute come discreta o cattiva, in confronto al $52.0 \%$ di quelli con disturbi organici cronici ed al $31.3 \%$ dei soggetti che non presentavano questo tipo di disturbi. Secondo il giudizio espresso dai medici di base, il $40.1 \%$ dei soggetti con disturbi psichici ed il $45.3 \%$ di quelli con disturbi organici cronici godevano di salute discreta o cattiva contro il $14.4 \%$ di quelli che non presentavano tali disturbi. Disabilità in campo occupazionale e nelle altre attività quotidiane è stata riportata dal $52.5 \%$ dei soggetti con disturbi psichici (nel $40.1 \%$ dei casi questa era moderata o grave), dal $44.4 \%$ dei soggetti con disturbi organici cronici (moderata o grave nel $26.8 \%$ dei casi) e dal $15.0 \%$ dei soggetti senza questi disturbi (moderata o grave nel $9.1 \%$ dei casi). Secondo il giudizio del ricercatore, il $48.4 \%$ dei soggetti con disturbi psichici, il $39.0 \%$ di quelli con disturbi organici cronici, ed il $27.6 \%$ di quanti non avevano questi disturbi presentavano disabilità. Il $60.0 \%$ dei soggetti con disturbi psichici lamentavano la presenza contemporanea di disturbi organici cronici; questi soggetti esibivano un peggior stato complessivo di salute e più alti livelli di disabilità rispetto a quanti presentavano esclusivamente disturbi psichici. Conclusioni - I disturbi psichici lamentati dai pazienti che si rivolgono ai servizi di medicina generale sono frequenti e costituiscono un importante problema di salute pubblica, comportando notevoli limitazioni per i pazienti ed elevati costi per la società, per cui si rendono opportuni appropriati programmi per il loro riconoscimento e trattamento.

Parole chiave: medicina generale, disturbi psichici, stato di salute, disabilità.

Summary. Objectives - To present the results obtained from a cross-sectional evaluation of a sample of primary care attenders selected in Verona in the framework of the World Health Organization International Multicentre Study on Psychological Problems in Primary Care Settings. Methods - Among consecutive attenders at 16 primary care clinics in Verona during the period April 1991/February 1992, a random sample, stratified on the basis of GHQ-12 scores, was selected for a thorough evaluation of psychological status, physical status and disability in occupational and other daily activities. All patients with psychopathological symptoms at baseline assessment and a $20 \%$ random sample of those without psychopathological symptoms were interviewed again after 3 and 12 months (data not presented here). Results Overall, 1,656 subjects were approached at the primary care clinics and 1,625 met inclusion criteria. The screening procedure was completed by 1,558 subjects and the second-stage evaluation by 250 . Psychiatric disorders according to ICD-10 criteria were diagnosed in $12.4 \%$ of consecutive primary care attenders; of these, about one-third $(4.5 \%$ of consecutive primary care attenders) satisfied ICD-10 diagnostic criteria for two or more disorders. Current Depressive Episode (4.7\%) and Generalized Anxiety Disorder (3.7\%) were the most common diagnoses. In addition, $11.2 \%$ of consecutive primary care attenders had 'sub-threshold' psychiatric disorders (i.e., they suffered from symptoms in at least two different areas among those listed in ICD-10, but they did not satisfy diagnostic criteria for well-defined disorders). Psychiatric disorders were more common among females and those aged 24-44 years. Only $20.6 \%$ of the subjects with psychiatric disorders contacted the general practitioner for their psychological symptoms, 5.7\% complained of symptoms which might have had a psychological origin, whereas in about $70 \%$ of the cases the psychiatric disorder was concealed behind the presentation of somatic symptoms, pains in various parts of the body or chronic physical illness. Sixty-two percent of the subjects with psychiatric disorders rated their health status as fair or poor, as compared to $52.0 \%$ of those with chronic physical illness and $31.3 \%$ of those without such disorders. According to the general practitioner, $40.1 \%$ of the subjects with psychiatric disorders and $45.3 \%$ of those with chronic physical illness had a fair or poor health status, compared to $14.4 \%$ of those without such disorders. Disability in occupational and other daily activities was reported by $52.5 \%$ of the subjects with psychiatric disorders (in $40.1 \%$ of the cases disability was moderate or severe), $44.4 \%$ of those with chronic physical illness (in $26.8 \%$ of the cases disability was moderate or severe), and $15.0 \%$ of the subjects without such disorders (in $9.1 \%$ of the cases disability was moderate or severe). According to the interviewer, disability was identified in $48.4 \%$ of the subjects with psychiatric disorders, $39.0 \%$ of those with chronic physical illness, and $27.6 \%$ of the subjects without such disorders. Sixty per cent of the subjects with psychiatric disorders suffered from concurrent chronic physical illness; these subjects had a poorer health status and higher disability levels than those with psychiatric disorders only. Conclusions - Psychiatric disorders among primary care attenders are frequent and represents a major public health problem, since they entail severe functional limitations for the patients and high costs for the society. Thus, appropriate programs for their recognition and treatment are needed.

Key words: primary health care, psychiatric disorders, chronic disease, health status, disability evaluation.

Ricevuto il 30.6.1994 - Revisione ricevuta il 13.9.94 - Accettato il 3.10.1994. 


\section{INTRODUZIONE}

Lo studio dei disturbi psichici tra i soggetti che si rivolgono ai servizi di medicina generale ha ricevuto crescente attenzione nel corso degli ultimi trent'anni e l'importanza del fenomeno è stata ripetutamente dimostrata a livello internazionale. È noto, infatti, che la maggior parte dei soggetti con disturbi psichici presenti nella popolazione generale sono visti ed eventualmente trattati nel contesto della medicina generale e solo una piccola percentuale giunge in contatto con i servizi psichiatrici. In Gran Bretagna, per esempio, la prevalenza annuale di disturbi psichici nell'ambito dei servizi di medicina generale è stata stimata intorno a $230 / 1.000$, dieci volte superiore rispetto a quella osservata a livello dei servizi psichiatrici considerati nel loro insieme (23.5/1.000) e quaranta volte più elevata rispetto a quella riscontrata nei servizi psichiatrici ospedalieri $(5.7 / 1.000)$ (Goldberg \& Huxley, 1992). Risultati sostanzialmente simili sono stati ottenuti considerando i tassi di consultazione per disturbi psichici, dal momento che questi ultimi, nell'ambito della medicina generale, superavano di circa 7 volte quelli relativi ai servizi psichiatrici ambulatoriali e di circa 60 volte i tassi di ricovero nei reparti psichiatrici ospedalieri (Sharp \& Morrel, 1989).

Il ruolo di primo piano che i servizi di medicina generale rivestono nel campo della salute mentale è stato riconosciuto anche in altri Paesi oltre alla Gran Bretagna. Utilizzando le stime di prevalenza dei disturbi psichici relative all'area di Verona-Sud, Tansella \& Williams (1989) hanno messo in evidenza che nel corso di una settimana i soggetti con disturbi psichici che si rivolgevano ai servizi di medicina generale erano circa 10 volte più numerosi rispetto a quelli in contatto con i servizi psichiatrici e 50 volte più numerosi rispetto a quanti venivano ricoverati nei reparti psichiatrici ospedalieri. A loro volta, i dati raccolti negli Stati Uniti nel corso dell'Epidemiologic Catchment Area Study (Robins \& Regier, 1991), un'indagine epidemiologica su larga scala condotta in un campione rappresentativo della popolazione nazionale, hanno rivelato che in un anno il $43.8 \%$ dei soggetti con disturbi psichici o legati all'abuso di sostanze avevano ricevuto trattamento nel contesto della medicina generale: si trattava di circa 10 milioni di soggetti, pari al $6.4 \%$ della popolazione nazionale (Narrow et al., 1993; Regier et al., 1993). Inoltre, considerando solamente le visite ambulatoriali effettuate negli Stati Uniti negli anni 1977 e 1978,
Rosenblatt et al. (1983) hanno dimostrato che i disturbi depressivi ed ansiosi erano all'origine del $3.1 \%$ di tutte le visite, collocandosi al quinto posto dopo le visite mediche generali (8.9\%), quelle per le infezioni respiratorie acute $(7.3 \%)$, per l'assistenza pre- e post-natale $(4.4 \%)$ e per l'ipertensione arteriosa $(4.4 \%)$; il $29 \%$ delle visite erano state effettuate nei servizi di medicina generale, il $12 \%$ nei servizi di medicina interna ed il $46 \%$ nei servizi psichiatrici, le rimanenti essendo distribuite in vari altri contesti.

Nonostante i servizi di medicina generale siano spesso organizzati in maniera diversa nei vari Paesi e di conseguenza il termine 'medicina generale' (primary care) riconosca accezioni e significati differenti, tuttavia sussiste un generale accordo sul fatto che tali servizi si trovano nella posizione migliore per identificare prontamente i soggetti sofferenti di un disturbo psichico presenti nella popolazione generale e per offrire trattamento a coloro che presentano questi disturbi: è noto, infatti, che i servizi di medicina generale rappresentano la sede di primo contatto con il sistema sanitario e sono in grado di fornire globalità e continuità di trattamenti non solo al singolo paziente, ma anche al suo nucleo familiare (WHO, 1978). Nel contesto della sua attività di diffusione e promozione della salute in generale, e di quella mentale in particolare, attraverso i servizi di medicina generale (WHO, 1990a), nel 1989 l'Organizzazione Mondiale della Sanità ha dato l'avvio ad uno studio internazionale multicentrico sui disturbi psichici nel contesto della medicina generale: tra le principali ragioni che hanno spinto ad intraprendere questo ambizioso programma, vi era la consapevolezza del fatto che in molti Paesi ancora mancavano dati relativi a tale problema oppure questi erano stati raccolti in tempi relativamente lontani, utilizzando criteri di campionamento e strumenti di valutazione non sempre idonei secondo i criteri attuali e prestando in genere scarsa attenzione al decorso ed all'esito a distanza di questo tipo di disturbi. A questo riguardo, altri progetti coordinati dall'Organizzazione Mondiale della Sanità avevano dimostrato che l'applicazione simultanea dello stesso protocollo d'indagine in Paesi molto diversi tra loro sul piano socio-economico e culturale non solo era attuabile dal punto di vista operativo, ma rappresentava anche una strategia utile e promettente per l'acquisizione di nuove conoscenze in una prospettiva trans-culturale (Sartorius et al., 1983; 1986; Gater et al., 1991).

Scopo del presente lavoro è quello di presentare alcuni dei risultati emersi dalla valutazione trasversale del campione di soggetti selezionato negli ambula- 
tori di medicina generale dell'area di Verona nell'ambito del suddetto studio internazionale multicentrico.

\section{METODO}

\section{A) Disegno dello studio}

Lo studio, coordinato dall'Organizzazione Mondiale della Sanità, si è svolto contemporaneamente in 15 Centri, situati in 14 Paesi diversi: Ankara in Turchia; Atene in Grecia; Bangalore in India; Berlino e Mainz in Germania; Groningen in Olanda; Ibadan in Nigeria; Manchester in Gran Bretagna; Nagasaki in Giappone; Parigi in Francia; Rio de Janeiro in Brasile; Santiago in Cile; Seattle negli Stati Uniti; Shanghai in Cina; Verona in Italia (Sartorius et al., 1993; Sartorius \& Üstün, in press; Piccinelli, 1994). In ciascuno di questi Centri è stato applicato lo stesso protocollo di ricerca quale è sintetizzato graficamente nella figura 1, nella quale è riportato anche il numero ottimale di soggetti da sottoporre a valutazione in ciascuna fase dell'indagine secondo le stime fornite dagli esperti dell'Organizzazione Mondiale della Sanità.

\section{I) Fase di screening}

Tra coloro che contattavano gli ambulatori di medicina generale, è stato stabilito di selezionare un campione randomizzato di soggetti di età compresa tra 15 e 65 anni; peraltro, molti Centri (tra questi anche Verona) hanno selezionato soggetti che avessero almeno 18 anni d'età, in grado quindi di decidere autonomamente se prendere parte o meno allo studio, essendo maggiorenni. L'esclusione dei soggetti di età superiore a 65 anni è stata decisa sulla base delle marcate differenze esistenti tra i vari Centri circa la frequenza delle classi di età più avanzate tra gli utenti dei servizi di medicina generale e della consapevolezza che tali differenze avrebbero ridotto la confrontabilità tra i campioni selezionati nei diversi Centri. Del pari sono stati esclusi gli individui troppo malati per completare gli strumenti previsti in questa fase (per esempio, pazienti psicotici); quanti avevano evidenti problemi di comunicazione (stranieri, per esempio); coloro che si erano rivolti al medico per un motivo non strettamente medico (certifi-
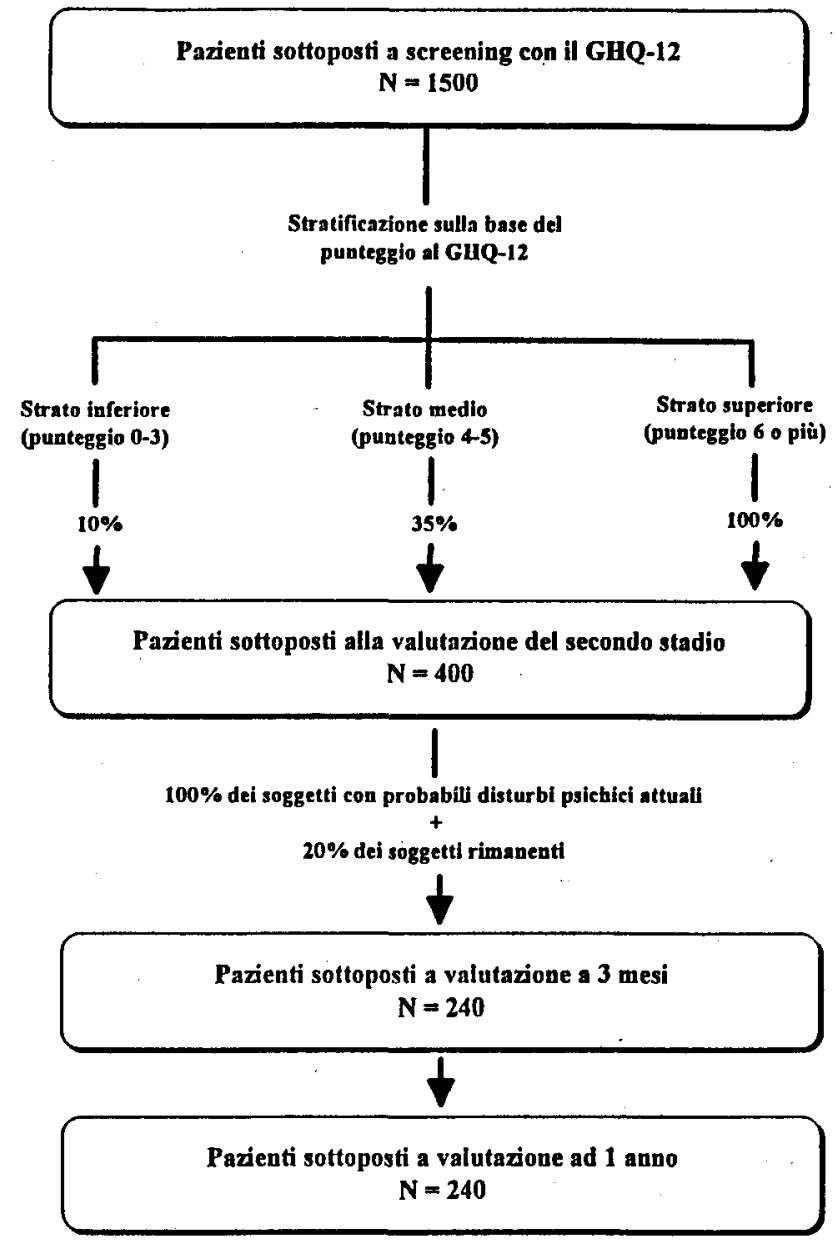

Figura 1. - Disegno dello studio.

cati per la patente, per esempio); e coloro che, non avendo fissa dimora, avrebbero potuto essere difficilmente ricontattati per la valutazione del secondo stadio. È stato inoltre stabilito che ciascun individuo fornisse dati una volta soltanto nel corso dello studio, per cui durante ogni singola sessione ambulatoriale sono stati esclusi $i$ soggetti che erano già stati sottoposti in precedenza alla procedura di screening.

Ai soggetti che soddisfacevano i criteri di inclusione nello studio sono stati spiegati gli scopi ed i requisiti dell'indagine e a quanti erano disposti a partecipare è stata fatta completare la versione a 12 domande del General Health Questionnaire (GHQ-12), un breve questionario di autovalutazione che indaga la presenza di disturbi psichici di natura non psicotica (Goldberg \& Williams, 1988). Sulla base del punteggio al GHQ-12, i soggetti che avevano completato il questionario sono stati assegnati ad uno di tre pos- 
sibili strati (inferiore, medio e superiore), i cui punteggi-soglia sono stati stabiliti in modo tale che circa il $60 \%$ dei soggetti cadessero nello strato inferiore (quello cioè con bassi punteggi), il $20 \%$ in quello medio ed il rimanente $20 \%$ nello strato superiore con i punteggi più elevati. A questo proposito, è importante ricordare che i punteggi-soglia tra i tre strati sono stati fissati specificamente per ciascun Centro, dal momento che nel corso di uno studio-pilota erano state riscontrate marcate differenze tra i vari Centri nella distribuzione dei punteggi riportati al GHQ-12. Se fossero stati impiegati identici punteggisoglia in tutti i Centri, la proporzione di soggetti selezionati per la valutazione del secondo stadio sarebbe stata notevolmente differente tra i Centri stessi: infatti, i Centri nei quali gli utenti dei servizi di medicina generale tendevano a riportare punteggi elevati al GHQ-12 avrebbero selezionato i circa 400 soggetti richiesti per la valutazione del secondo stadio dopo aver sottoposto a screening un numero considerevolmente inferiore di utenti, creando in tal modo una situazione logisticamente e scientificamente inappropriata.

Una volta fissati in questo modo i tre strati, è stato stabilito di selezionare in maniera randomizzata da ciascuno strato una quota fissa di soggetti da sottoporre alla valutazione del secondo stadio (il $100 \%$ dei soggetti assegnati allo strato superiore, il $35 \%$ di quelli inclusi nello strato medio ed il $10 \%$ dei soggetti che figuravano nello strato inferiore), affinché i soggetti così selezionati avessero un'elevata probabilità di presentare disturbi psichici alla successiva intervista psichiatrica e non fosse compromessa al tempo stesso la possibilità di ottenere stime accurate della frequenza dei disturbi psichici riferite al campione originale di pazienti sottoposti inizialmente a screening. Come risulta dalla figura 1 , i punteggi-soglia impiegati a Verona per $i$ tre strati sono stati rispettivamente quelli di 4 e 6 : i soggetti con punteggio al GHQ-12 minore di 4 sono stati così assegnati allo strato inferiore, quelli con punteggio 4 o 5 allo strato medio, e quelli con punteggio uguale o superiore a 6 allo strato superiore.

La procedura di campionamento è stata realizzata grazie ad appositi moduli (Modulo di Campionamento) forniti dall'Organizzazione Mondiale della Sanità, sui quali, oltre allo spazio per registrare i dati anagrafici del paziente, figurava il punteggio-soglia che questi avrebbe dovuto uguagliare o superare al GHQ-12 per poter essere selezionato per la valutazione del secondo stadio. Questi moduli sono stati concepiti grazie all'impiego di procedure compute- rizzate, tali per cui il campione finale sottoposto alla valutazione del secondo stadio provenisse dai tre strati identificati sulla base del punteggio al GHQ-12 secondo le percentuali riportate in precedenza. Infine, per ciascun soggetto selezionato per la valutazione del secondo stadio il medico di base registrava su di un'apposita scheda (Scheda del Medico) il motivo del contatto, il livello complessivo di salute, la gravità di un'eventuale disturbo di tipo fisico e/o psichico e, per quest'ultima eventualità, la diagnosi e l'eventuale trattamento prescritto; infine, venivano riportate le tre diagnosi più importanti (a giudizio del medico di base) che figuravano nella cartella clinica del paziente.

\section{II) Valutazione del secondo stadio}

Entro 10 giorni dal momento della selezione avvenuta nell'ambulatorio del medico di base è stata condotta da un ricercatore la valutazione del secondo stadio. A Verona questa ha avuto luogo in forma rigorosamente personale e privata a casa del paziente oppure presso il Servizio di Psicologia Medica dell'Istituto di Psichiatria dell'Università di Verona; in quest'ultima eventualità è stata offerta al paziente una somma di denaro per coprire le spese di viaggio. Gli strumenti utilizzati nel corso della valutazione del secondo stadio comprendevano:

1) la Versione per la Medicina Generale della Composite International Diagnostic Interview (CIDI-PHC), che è stata sviluppata per questo studio a partire dalla Composite International Diagnostic Interview (CIDI) (Robins et al., 1988) e, tradotta in 13 lingue diverse, ha dimostrato di poter essere somministrata da intervistatori con o senza una specifica preparazione nel campo della salute mentale con buone caratteristiche di riproducibilità (come testimoniato dai valori di kappa di Cohen compresi tra 0.82 e 1.00 a seconda delle diverse domande dell'intervista) (WHO, 1990b). Questi risultati concordano con quelli riportati da uno studio internazionale multicentrico che è stato condotto in 20 diverse nazioni per valutare la riproducibilità inter-intervistatore degli items della CIDI e delle diagnosi da essa derivate e che ha evidenziato valori di kappa di Cohen molto elevati, con buon accordo tra intervistatori clinici e non clinici (Tacchini et al., 1994).

In particolare, la CIDI-PHC comprende: a) le sezioni della CIDI relative alla somatizzazione, l'ipocondria, la nevrastenia, i disturbi d'ansia e quelli depressivi, opportunamente modificate per permettere 
di valutare lo stato psicopatologico del soggetto intervistato sia riguardo ad eventuali disturbi lamentati lungo il corso della vita (diagnosi lifetime) sia durante il mese precedente l'intervista; b) una sezione dedicata all'uso di bevande alcooliche, che include domande tratte dall'Alcohol Use Disorder Identification Test (Babor et al., 1989), per indagare l'eventuale presenza di disturbi legati all'abuso di alcool secondo i criteri dell'ICD-10; c) una sezione per la valutazione delle capacità cognitive del paziente, in gran parte ispirata al Mini Mental State (Folstein et al., 1975); d) domande aggiuntive rispetto alla CIDI, in grado di esplorare la presenza di alcuni sintomi frequentemente lamentati dai soggetti che si rivolgono ai servizi di medicina generale (per esempio, sintomi dispeptici, acufeni, parestesie, tremori, ecc.); e) domande rivolte al paziente e relative alla ragione della consultazione, alla possibile presenza di comuni condizioni organiche croniche (per esempio, malattie cardio-circolatorie, diabete, tumori, epilessia, asma bronchiale, ecc.), all'assunzione di farmaci nel corso del mese precedente l'intervista ed alla eventuale coesistenza ed ai rapporti intercorrenti tra disturbi psicopatologici e disturbi organici; f) una sezione in grado di ricostruire il percorso seguito da ciascun paziente all'interno delle strutture sanitarie (pubbliche e private) prima di giungere in contatto con il proprio medico di base;

2) la Social Disability Schedule (Wiersma et al., 1988), un'intervista semi-strutturata che valuta il grado di disabilità sociale di un soggetto tenendo conto delle diverse aspettative legate allo specifico contesto culturale di appartenenza. Nel presente studio sono state utilizzate soltanto le sezioni dell'intervista relative al ruolo occupazionale ed alle altre attività quotidiane. L'accordo tra intervistatore ed osservatore, espresso dall'indice kappa, è risultato compreso tra 0.72 e 0.93 a seconda dei singoli items;

3) il Brief Disability Questionnaire, un questionario di autovalutazione, che, sviluppato a partire dalla World Health Organization Disability Assessment Schedule (WHO, 1980) e dal Medical Outcome Survey Short Form-36 (Stewart et al., 1988; Ware \& Sherbourne, 1992), indaga il livello di disabilità del soggetto nelle proprie attività abituali;

4) una scheda (Scheda sulla Salute) per la valutazione del livello complessivo di salute secondo il giudizio espresso dal paziente su di una scala a 5 categorie di gravità crescente (comprese tra 'eccellente' e 'cattivo').

5) la versione a 28 domande del General Health Questionnaire (GHQ-28) (Goldberg \& Williams,
1988), che permette di ottenere, oltre ad un punteggio complessivo indicativo del livello di disagio emotivo del paziente, anche punteggi distinti relativi a 4 sottoscale (sintomi somatici; ansia ed insonnia; disabilità sociale; depressione grave).

\section{III) Valutazione longitudinale}

A distanza di tre mesi e poi di un anno dal giorno della valutazione iniziale sono stati invitati ad essere nuovamente contattati da un ricercatore tutti i soggetti che in almeno una delle sezioni della CIDIPHC avessero superato una soglia prefissata basata sul conteggio dei sintomi lamentati (per esempio, 4 sintomi neuro-vegetativi legati all'ansia, oppure 4 sintomi depressivi, e cosi via) e che quindi presentassero verosimilmente un disturbo psichico in atto; a questi è stato aggiunto un campione di soggetti selezionato in maniera randomizzata e costituito dal $20 \%$ di coloro che non soddisfacevano tale criterio. A tre mesi è stata prevista una breve valutazione nel corso della quale sono stati fatti completare al paziente il $G H Q-28$, il Brief Disability Questionnaire e la Scheda sulla Salute; inoltre, sono state raccolte informazioni sull'assunzione di farmaci e sulla consultazione dei servizi sanitari nel corso dei tre mesi successivi alla valutazione del secondo stadio, grazie all'impiego di appositi questionari (Medication Use Questionnaire ed Health Care Use Questionnaire). Nella valutazione effettuata a distanza di un anno sono stati impiegati gli stessi strumenti previsti per la valutazione del secondo stadio con l'aggiunta del Medication Use Questionnaire e del Health Care Use Questionnaire, opportunamente modificati per indagare il decorso dei sintomi lamentati dal paziente, l'assunzione di farmaci e la consultazione dei servizi sanitari nel corso dell'anno successivo alla valutazione iniziale.

La fase di screening si è protratta tra l'aprile 1991 ed il febbraio 1992, ma è stata interrotta nei mesi di luglio ed agosto dal momento che il numero di soggetti che si rivolgevano agli ambulatori di medicina generale e la loro disponibilità per la valutazione del secondo stadio erano piuttosto ridotti a causa delle vacanze estive; come previsto dal protocollo, le due valutazioni longitudinali sono state effettuate a distanza di tre mesi ed un anno circa da quella iniziale. In totale, 8 medici, una psicologa, due assistenti sanitarie, un'assistente sociale ed un'infermiera professionale sono stati variamente coinvolti nel corso dello studio. Gli strumenti previsti dalla valutazione del 
secondo stadio e dalle due valutazioni longitudinali sono stati somministrati esclusivamente da personale con formazione clinica nel campo della salute mentale (medici e psicologa), mentre la procedura di screening è stata di preferenza affidata al personale 'non clinico'.

\section{B) Numerosità campionaria}

Tra i requisiti dello studio era stato stabilito che i dati raccolti a livello di ogni singolo Centro avrebbero dovuto permettere di produrre stime accurate della frequenza dei disturbi psichici tra $\mathbf{i}$ soggetti selezionati presso jl Centro stesso. Inoltre, la numerosità del campione di soggetti inclusi nello studio avrebbe dovuto offrire a ciascun Centro la possibilità di indagare localmente le differenze esistenti tra diversi gruppi di disturbi psichici (per esempio, disturbi che soddisfacevano in tutto $o$ in parte $i$ criteri diagnostici previsti dagli attuali sistemi di classificazione; disturbi riconosciuti o non riconosciuti dal medico di base) potendo contare su di un'adeguata 'forza' statistica; parimenti, due diversi Centri avrebbero dovuto avere la possibilità di individuare come statisticamente significative eventuali differenze intercorrenti tra di essi per quanto riguarda quei disturbi la cui frequenza tra gli utenti dei servizi di medicina generale fosse pari almeno al $5 \%$. I calcoli presuntivi eseguiti prima dell'inizio dello studio dagli esperti dell'Organizzazione Mondiale della Sanità hanno indicato che per soddisfare tali requisiti si rendeva necessario contattare presso ciascun Centro all'incirca 1.700 soggetti tra quanti si rivolgevano consecutivamente ai servizi di medicina generale; di questi, 1.500 circa avrebbero dovuto completare la procedura di screening e 400 la valutazione del secondo stadio; infine, per circa 240 soggetti avrebbero dovuto essere completate le due valutazioni effettuate a distanza di tre mesi ed un anno rispettivamente.

\section{C) Costi}

Sulla base del numero ottimale di soggetti da sottoporre a valutazione, per ciascuna fase dell'indagine sono stati calcolati $i$ costi presuntivi corrispondenti, aggiornati al maggio 1994. È importante tenere presente che si tratta di una stima per difetto, in quanto non sono stati considerati: a) le spese comportate dalla traduzione degli strumenti dall'inglese in italiano e dalla successiva back-translation; questa scelta è stata motivata dal fatto che gli strumenti in lingua italiana sono attualmente a disposizione dei ricercatori che ne volessero fare uso, dietro richiesta scritta ad uno degli autori (M.T.); b) i costi necessari per addestrare i ricercatori all'uso degli strumenti utilizzati nel corso dello studio'; c) il tempo che si è reso necessario per richiedere la collaborazione dei medici di base e per spiegare loro i presupposti dell'indagine e le modalità d'impiego degli strumenti di loro pertinenza; d) le spese inerenti il viaggio per raggiungere gli ambulatori di medicina generale e le abitazioni dei pazienti da sottoporre a valutazione, in quanto ampiamente variabili e difficilmente quantificabili; e) il costo del programma di inserimento dei dati, essendo stato fornito gratuitamente dall'Organizzazione Mondiale della Sanità ai Centri partecipanti ed essendo anch'esso disponibile su richiesta ad uno degli autori (T.B.Ü.); f) infine, non sono stati inclusi i costi necessari per l'analisi dei dati raccolti nel corso dello studio in vista della loro pubblicazione (analisi statistica, interpretazione e commento dei dati, preparazione del dattiloscritto); si tratta di costi difficilmente quantificabili, ma sicuramente elevati.

Sono state considerate, invece:

a) le spese legate al materiale cartaceo. A tal fine, sono state contattate telefonicamente tre copisterie site in Verona ed è stato poi scelto il prezzo intermedio tra quelli riferiti, che è risultato pari a lire 90 per foglio;

b) le spese richieste dal lavoro di raccolta dei dati. È stato stabilito di affidare idealmente ad un assistente sanitario la procedura di screening e la valutazione prevista a distanza di tre mesi, in quanto effettuabili anche da personale senza formazione medica. Al contrario, la somministrazione della CIDI-PHC e degli altri strumenti previsti dalla valutazione del secondo stadio e da quella effettuata a distanza di un anno è stata affidata idealmente ad un assistente psichiatra, in considerazione del fatto che, sebbene la CIDI fosse stata originariamente concepita per poter essere somministrata anche da personale senza formazione specifica nel campo della salute mentale, le modifiche apportate per ottenere la CIDI-PHC rendono consigliabile l'impiego di personale con formazione medica. Sulla base dello stipendio annuale

\footnotetext{
1 Presso il Servizio di Psicologia Medica vengono organizzati, dietro richiesta scritta inviata ad uno degli autori (M.T.), corsi di addestramento all'uso della Versione per la Medicina Generale della Composite International Diagnostic Interview (CIDI-PHC). Il costo si aggira intorno a lire 400.000 per partecipante.
} 
comprensivo di tutti gli oneri per l'Ente è stato calcolato il costo orario per un assistente sanitario ed un assistente psichiatra, che è risultato pari a lire 23.660 e 36.840 , rispettivamente.

Per la procedura di screening si è assunto che il medico di base visitasse in media 4 pazienti all'ora. La durata media della valutazione del secondo stadio è stata invece stimata sulla base dei tempi impiegati per completare le prime 25 e le ultime 25 valutazioni effettuate nel corso della nostra indagine ed è risultata pari a 70.5 minuti; sono stati poi aggiunti altri 20 minuti necessari per la codifica delle diagnosi riportate dal paziente nel corso dell'intervista secondo i codici dell'ICD-10, per cui la durata totale della valutazione del secondo stadio è stata fissata a $90 \mathrm{mi}-$ nuti. Lo stesso tempo è stato ritenuto necessario anche per la valutazione effettuata a distanza di un anno, mentre per la valutazione effettuata a distanza di tre mesi è stata fissata una durata di 20 minuti. Infine, la raccolta dei dati da parte del medico di base è stata ritenuta priva di costo, in quanto abitualmente inserita nella sua attività di routine;

c) le spese conseguenti all'inserimento dei dati nel calcolatore. Per la loro determinazione, sono state calcolate per ciascuna fase dell'indagine le battute minime e massime richieste a seconda che un soggetto non presentasse alcun sintomo tra quelli indagati oppure li presentasse tutti ed è stata poi effettuata la media tra i due valori. Il prezzo per battuta è stato stabilito dopo aver contattato telefonicamente tre centri per l'elaborazione dei dati in Verona e scegliendo il prezzo intermedio tra quelli riferiti, che è risultato pari a lire 5 .

I costi così stimati sono riportati nella tabella $\mathrm{I}$.

A questi costi vanno aggiunti quelli per eventuali rifiuti a partecipare all'indagine, dal momento che $i$ soggetti che rifiutano dovrebbero essere rimpiazzati $\mathrm{da}$ altri per raggiungere il numero finale di $400 \mathrm{sog}$ getti sottoposti alla valutazione del secondo stadio. Tali costi (materiale cartaceo + raccolta dei dati + inserimento dei dati) sono stati stimati a lire 6.505 per ciascun soggetto che rifiuta di sottoporsi alla procedura di screening e a lire 8.105 per ciascun soggetto che si sottopone alla procedura di screening, ma rifiuta poi la valutazione del secondo stadio; anche coloro che, già selezionati in una fase precedente

Tabella I. - Costi indicativi dello studio aggiornati a maggio 1994 (vedi testo).

\begin{tabular}{|c|c|c|c|c|c|c|c|}
\hline & \multicolumn{2}{|c|}{ Materiale } & \multicolumn{2}{|c|}{ Raccolta dati } & \multicolumn{2}{|c|}{ Inserimento dati } & \multirow[b]{2}{*}{$\begin{array}{l}\text { Totale costi } \\
\text { (lire) }\end{array}$} \\
\hline & $\begin{array}{l}\text { Fogli } \\
(\mathrm{N})\end{array}$ & $\begin{array}{l}\text { Costo } \\
\text { (lire) }\end{array}$ & $\begin{array}{l}\text { Ore } \\
(\mathrm{N})\end{array}$ & $\begin{array}{l}\text { Costo } \\
\text { (lire) }\end{array}$ & $\begin{array}{l}\text { Battute } \\
(\mathrm{N})\end{array}$ & $\begin{array}{l}\text { Costo** } \\
\text { (lire) }\end{array}$ & \\
\hline $\begin{array}{l}\text { Screening } \\
(\mathrm{N}=1500)\end{array}$ & $\begin{array}{l}2 \\
1^{*}\end{array}$ & $\begin{array}{c}270.000 \\
18.000^{*}\end{array}$ & $\begin{array}{c}375 \\
50^{*}\end{array}$ & $\begin{array}{l}8.872 .500 \\
1.183 .000^{*}\end{array}$ & $\begin{array}{l}66 \\
50^{*}\end{array}$ & $\begin{array}{l}990.000 \\
100.000^{*}\end{array}$ & $\begin{array}{c}10.132 .500 \\
1.301 .000^{*}\end{array}$ \\
\hline $\begin{array}{l}\text { Secondo stadio } \\
(\mathrm{N}=400)\end{array}$ & 64 & 2.304 .000 & 600 & 22.104 .000 & 1.048 & 4.192 .000 & 28.600 .000 \\
\hline $\begin{array}{l}\text { Follow-up ( } 3 \text { mesi) } \\
(\mathrm{N}=240)\end{array}$ & 6 & 129.000 & 80 & 1.892 .800 & 144 & 345.600 & 2.367 .400 \\
\hline $\begin{array}{l}\text { Follow-up (1 anno) } \\
(\mathrm{N}=240)\end{array}$ & 57 & 1.231 .200 & 360 & 13.262 .400 & 869 & 2.085 .600 & 16.579 .200 \\
\hline $\begin{array}{l}\text { Dati forniti dal medico } \\
(\mathrm{N}=400)\end{array}$ & 4 & 144.000 & na & na & 99 & 396.000 & 540.00 \\
\hline Totale & 134 & 4.096 .200 & 1.465 & 47.314 .700 & 2.276 & 8.109 .200 & 59.520 .100 \\
\hline
\end{tabular}

* Pazienti non selezionabili per lo studio $(\mathrm{N}=200)$

** I costi sono moltiplicati per 2 essendo stati introdotti i dati nel computer due volte per verificare eventuali errori di battitura na $=$ non applicabile

Nota. In questa tabella non sono inclusi i costi per tradurre gli strumenti, per addestrare i ricercatori all'uso degli strumenti e per richiedere la collaborazione dei medici di medicina generale, le spese di viaggio per raggiungere gli ambulatori di medicina generale, il costo del programma per l'inserimento dei dati, i costi per l'analisi dei dati, l'interpretazione dei risultati e la preparazione del dattiloscritto. 
dell'indagine, si ripresentano nuovamente dal medico durante lo svolgimento dello studio, comportano un costo aggiuntivo pro capite di lire 6.505. Al contrario, i soggetti che rifiutano una od entrambe le valutazioni longitudinali sono soggetti persi per lo studio e, al di là dei costi necessari per ricontattarli (già considerati nella tabella I), non comportano altri costi. Ammettendo dunque un tasso di rifiuto alla procedura di screening pari al $5 \%$ ed alla valutazione del secondo stadio pari al $30 \%$ (percentuali medie calcolate combinando i dati forniti da tutti i Centri partecipanti allo studio internazionale descritto in questa sede) ed un 'ricircolo' di pazienti pari al $10 \%$ durante un periodo di 12 settimane (durata dello studio consigliata dall'Organizzazione Mondiale della Sanità), è necessario considerare un costo supplettivo di lire 2.436.225. Inoltre, i costi per contattare telefonicamente i pazienti a distanza di tre mesi e poi di un anno da parte di un assistente sanitario (1 scatto per paziente, pari a lire 127 , per 5 minuti di conversazione) sono stati stimati a lire 1.007.360. Infine, il costo del lavoro di coordinamento (compilazione dei registri, archiviazione del materiale, ecc.), affidato ad un assistente sanitario e fissato a circa 8 ore settimanali durante le 12 settimane di durata dello studio, può essere stimato in lire 2.271.360. In totale, quindi, il costo stimato dello studio può essere fissato a lire 65.235.045.

\section{D) Selezione degli ambulatori di medicina generale a Verona}

Gli ambulatori di medicina generale inclusi nell'indagine condotta a Verona sono stati selezionati sulla base della disponibilità dei rispettivi medici. Sebbene la selezione degli ambulatori non sia avvenuta in maniera randomizzata e non sia perciò possibile affermare che essi siano rappresentativi in senso stretto della totalità degli ambulatori di medicina generale presenti nell'area di Verona, tuttavia non ci sono elementi per ritenere che essi differissero in misura significativa dalla media degli ambulatori situati nell'area geografica considerata, dal momento che questo costituiva espressamente il principale criterio ai fini dell'esclusione di un ambulatorio dallo studio. In totale, all'indagine hanno partecipato 16 medici di base, che nel complesso garantivano la loro assistenza a circa 20.000 soggetti. Sebbene alcuni medici condividessero le stesse strutture ambulatoriali, tuttavia ciascun medico aveva una lista distinta dei pro- pri pazienti; tutti gli ambulatori, tranne uno, erano sprovvisti di personale infermieristico

L'indagine è stata inizialmente condotta nell'area di Verona-Sud, che comprende la parte meridionale della città di Verona e tre piccoli comuni limitrofi (Buttapietra, Cadidavid e Castel d'Azzano); nel corso degli ultimi mesi della fase di screening, nello sforzo di aumentare il numero di pazienti da sottoporre a selezione, sono stati inclusi anche 5 medici di base i cui ambulatori erano situati in Verona o provincia, ma al di fuori dell'area di Verona-Sud.

L'area di Verona-Sud si estende per circa $76 \mathrm{Km}^{2}$ ed ha una popolazione di circa 75.000 abitanti; i residenti sono impiegati principalmente nel settore dei servizi $(\mathbf{5 7 . 4 \% )}$ ) e nell'industria $(38.8 \%)$, mentre l'agricoltura gioca solo un ruolo marginale nell'economia locale; il tasso di disoccupazione, calcolato come percentuale della popolazione attiva di età uguale o superiore a 14 anni, si aggira intorno all' $8 \%$ (dati ricavati dal censimento del 1981). Tale area è stata inizialmente scelta perché costituisce il bacino d'utenza del Servizio Psichiatrico Territoriale diretto dall'Istituto di Psichiatria dell'Università di Verona e perché fin dalla sua istituzione nel 1979 il Registro Psichiatrico dei Casi di Verona-Sud fornisce informazioni su tutti i contatti psichiatrici avuti dai residenti con i servizi specialistici situati nella provincia di Verona (Balestrieri et al., 1992). Ciò rappresentava un indubbio vantaggio, in quanto permetteva di integrare $\mathrm{i}$ dati raccolti nel contesto della medicina generale con quelli disponibili a livello dei servizi specialistici. Inoltre, nel corso di precedenti ricerche condotte negli ambulatori di medicina generale situati a Verona-Sud si erano stabiliti legami di mutua collaborazione tra i rispettivi medici di base e la nostra Unità di ricerca, che è parte dell'Istituto di Psichiatria.

\section{E) Materiale}

La diagnosi di disturbo psichico è stata formulata attraverso la somministrazione della CIDI-PHC, facendo riferimento ai criteri nosografici dell'ICD-10 (WHO, 1992). In particolare, sono state considerate le seguenti categorie diagnostiche: Episodio Depressivo (F32/33.xx); Distimia (F34.1); Agorafobia (F40.0); Sindrome da Attacchi di Panico (F41.0); Sindrome Ansiosa Generalizzata (F41.1); Sindrome di Somatizzazione (F45.0); Sindrome Ipocondriaca (F45.2); Nevrastenia (F48.0); Dipendenza da Alcool (F10.2); Uso Dannoso di Alcool (F10.1). 
La valutazione relativa allo stato complessivo di salute è stata raccolta dai pazienti e dai rispettivi medici di base, grazie ad una semplice scala a 5 categorie di gravità crescente, comprese tra 'eccellente' e 'cattivo'.

Il giudizio del paziente relativo al proprio grado di disabilità è stato ricavato dalle risposte fornite al Brief Disability Questionnaire. Delle 13 domande di cui è composto il questionario, le prime 11 indagano la frequenza e la gravità delle limitazioni occorse al paziente in varie attività quotidiane svolte nel corso dell'ultimo mese; per esse il paziente può scegliere tra tre possibili risposte a seconda che la limitazione non si sia verificata (punteggio 0 ) oppure si sia verificata solo qualche volta (1) o con maggiore frequenza (2). Le ultime due domande si riferiscono al numero di giorni durante i quali il paziente è stato completamente incapace di svolgere le sue attività quotidiane oppure è rimasto a letto per la maggior parte del giorno. Al fine di ottenere 4 categorie di disabilità crescente, sono state considerate solo le prime 11 domande del questionario ed il punteggio totale risultante è stato ricodificato nel modo seguente: nessuna disabilità (punteggio tra 0 e 4); disabilità lieve (5-7); disabilità moderata (8-13); disabilità grave (14-22).

Il ricercatore ha espresso il proprio giudizio sulla disabilità sofferta nell'ultimo mese dal paziente in ambito lavorativo e nelle altre attività quotidiane, grazie all'impiego del modulo corrispondente incluso nella Social Disability Schedule. Con questo strumento, per ciascun soggetto vengono valutati 4 aspetti distinti (routine lavorativa, performance nelle attività lavorative, rapporti con altre persone al lavoro, impiego del tempo libero), dalla cui combinazione è possibile assegnare un punteggio totale di disabilità compreso tra 0 (nessuna disabilità) e 3 (disabilità grave); fanno eccezione le casalinghe, per le quali non si considerano, per ovvi motivi, i rapporti con altre persone al lavoro, ed i disoccupati ed i pensionati, per i quali si considera solo l'impiego del tempo libero.

\section{F) Analisi statistiche}

I soggetti sottoposti alla valutazione del secondo stadio sono stati selezionati in maniera randomizzata utilizzando una tecnica di campionamento stratificato basata sul punteggio riportato al GHQ-12 (come descritto in precedenza). Tale procedura di campionamento è stata tenuta in considerazione nel mo- mento in cui i dati raccolti durante la valutazione del secondo stadio sono stati riferiti al campione originale di soggetti sottoposti inizialmente a screening: ciascun soggetto aveva infatti una diversa probabilità di essere selezionato per la valutazione del secondo stadio a seconda del punteggio riportato al GHQ-12, essendo tale probabilità maggiore per i soggetti che avevano ottenuto punteggi elevati. Per ciascun soggetto incluso nella valutazione del secondo stadio è stato perciò calcolato un apposito coefficiente (o weight, alla lettera 'peso') in grado di tener conto non solo della diversa probabilità di selezione, ma anche del diverso tasso di rifiuto riscontrato nel corso dello studio in rapporto al sesso dei soggetti ed al punteggio riportato al GHQ-12. Tali coefficienti sono stati calcolati separatamente per ciascun sesso e per ciascuno dei tre strati basati sul punteggio al GHQ-12, in modo tale che tutti i soggetti dello stesso sesso ed appartenenti allo stesso strato di punteggio del GHQ-12 avessero anche identici coefficienti. Tali coefficienti sono stati così impiegati per 'pesare' i dati raccolti nel corso della valutazione del secondo stadio ed ottenere stime di frequenza accurate riferite al campione di soggetti che erano stati sottoposti inizialmente a screening.

Ciascun coefficiente relativo ad un dato gruppo di soggetti identificato sulla base delle due variabili sesso e punteggio al GHQ-12 è stato calcolato dividendo il numero di soggetti sottoposti inizialmente a screening per quello dei soggetti che avevano completato la valutazione del secondo stadio ed appartenevano al gruppo stesso. Per esempio, se 200 maschi con punteggio al GHQ-12 inferiore a 4 erano stati sottoposti a screening e di questi 18 avevano completato la valutazione del secondo stadio, il corrispondente coefficiente era pari a $11.1(200 / 18)$. Dal momento che nel computo delle stime di frequenza tramite i comuni programmi statistici per computer era più agevole tenere al denominatore il numero totale di soggetti che avevano completato la valutazione del secondo stadio (piuttosto che quello dei soggetti sottoposti inizialmente a screening), i coefficienti cosi ottenuti sono stati moltiplicati per il valore del rapporto tra il numero totale di soggetti intervistati nel corso della valutazione del secondo stadio e quello dei soggetti sottoposti inizialmente a screening.

Nella sezione relativa ai Risultati verranno presentati, accanto ai dati non 'pesati' relativi al campione di soggetti sottoposti alla valutazione del secondo stadio, anche le stime ponderate ottenute per la variabile in questione sulla base dei coefficienti testè descritti e riferite perciò al campione originale di 
soggetti sottoposti inizialmente a screening. Le stime di frequenza riportate rappresentano dunque dati patient-based e non dati population-based, non si tratta cioè di tassi calcolati rispetto alla popolazione che risiede nell'area geografica considerata e che consulta i servizi di medicina generale.

\section{RISULTATI}

In totale, 1.656 soggetti sono stati contattati dai ricercatori negli ambulatori di medicina generale e, di questi, 1.625 soddisfacevano i requisiti richiesti per essere inclusi nello studio. La procedura di screening è stata completata da 1.558 soggetti $(95.9 \%$ del campione selezionato); tra i 457 soggetti selezionati per la valutazione del secondo stadio, $250(54.7 \%)$ hanno condotto a termine la valutazione, 3 hanno interrotto prematuramente l'intervista ed i rimanenti hanno rifiutato di essere intervistati o non hanno rispettato gli appuntamenti fissati. Tra le principali ragioni avanzate a sostegno del rifiuto sono state menzionate soprattutto la mancanza di tempo e lo scarso interesse per l'indagine, presumibilmente in rapporto col fatto che ai soggetti selezionati era stata comunicata l'impossibilità di ricevere trattamento per gli eventuali disturbi presentati.

\section{Caratteristiche socio-demografiche}

Nella tabella II sono riportate le caratteristiche socio-demografiche del campione di soggetti inclusi nello studio.

Due terzi circa dei soggetti erano di sesso femminile; il gruppo di età più rappresentato era quello compreso tra 45 e 65 anni; la maggior parte dei soggetti erano coniugati. Poco meno della metà dei soggetti avevano completato fino a 8 anni di scuola, lasciando quindi la scuola non appena raggiunta l'età minima richiesta; solo una minoranza avevano continuato i propri studi oltre la scuola media superiore $o$ stavano ancora frequentando la scuola al momento dell'indagine. Poco più della metà dei soggetti avevano un lavoro retribuito; le casalinghe contavano per circa un terzo del campione, sebbene tale percentuale si riferisca a quegli individui che svolgevano unicamente le faccende domestiche e non avevano contemporaneamente alcun genere di lavoro retribuito (anche a tempo definito); infine, i soggetti disoc-
Tabella II. - Caratteristiche socio-demografiche.

\begin{tabular}{|c|c|c|c|}
\hline & \multicolumn{2}{|c|}{ Pazienti intervistati } & \multirow[t]{2}{*}{ Stime ponderate } \\
\hline Sesso & & & \\
\hline Maschi & 83 & 33.2 & 36.2 \\
\hline Femmine & 167 & 66.8 & 63.8 \\
\hline \multicolumn{4}{|l|}{ Età } \\
\hline $18-24$ & 28 & 11.2 & 12.0 \\
\hline 25.44 & 104 & 41.6 & 41.2 \\
\hline $45-65$ & 108 & 47.2 & 46.8 \\
\hline \multicolumn{4}{|l|}{ Anni di scuola } \\
\hline $1-5$ & 69 & 27.6 & 29.8 \\
\hline $6-8$ & 62 & 24.8 & 17.8 \\
\hline $9-13$ & 95 & 38.0 & 45.6 \\
\hline 14 o più & 17 & 6.8 & 5.3 \\
\hline Studente & 5 & 2.0 & 1.0 \\
\hline Non conosciuto & 2 . & 0.8 & 0.4 \\
\hline \multicolumn{4}{|l|}{ Stato civile } \\
\hline Mai sposato & 52 & 20.8 & 18.7 \\
\hline Sposato & 166 & 66.4 & 70.7 \\
\hline Separato/divorziato & 16 & 6.4 & 5.7 \\
\hline Vedovo & 15 & 6.0 & 4.6 \\
\hline Non conosciuto & 1 & 0.4 & 0.3 \\
\hline \multicolumn{4}{|l|}{ Occupazione } \\
\hline Occupato & 134 & 53.6 & 51.5 \\
\hline Disoccupato & 9 & 3.6 & 2.2 \\
\hline Casalinga & 71 & 28.4 & 30.3 \\
\hline Studente & 5 & 2.0 & 1.9 \\
\hline Pensionato & 27 & 10.8 & 12.4 \\
\hline Non conosciuto & 4 & 1.6 & 1.6 \\
\hline
\end{tabular}

cupati $(2.2 \%)$ erano presenti in percentuale inferiore rispetto al tasso di disoccupazione relativo all'area di Verona-Sud $(8.4 \%)$.

Frequenza dei disturbi psichici

Come riportato nella tabella III, il $12.4 \%$ dei soggetti che avevano contattato gli ambulatori di medicina generale presentava disturbi psichici ben definiti secondo i criteri dell'ICD-10 e, di questi, un terzo circa $(4.5 \%$ dei soggetti sottoposti inizialmente a screening) soddisfaceva i criteri diagnostici per la presenza contemporanea di due o più disturbi psichici diversi tra quelli considerati nel presente studio (co-morbidity). La diagnosi di Episodio Depressivo Attuale $(4.7 \%)$ e quella di Sindrome Ansiosa Generalizzata $(3.7 \%)$ sono risultate le più frequenti, se- 
Tabella III. - Prevalenza dei disturbi psichici attuali secondo l'ICD-10.

\begin{tabular}{lrrc}
\hline Categoria diagnostica & $\begin{array}{c}\text { Pazienti } \\
\text { intervistati }\end{array}$ & $\begin{array}{c}\text { Stime } \\
\text { ponderate }\end{array}$ \\
& $\mathrm{N}$ & \multicolumn{1}{c}{$\%$} & $\%$ \\
\hline Dipendenza da alcool (F10.2) & 3 & 1.2 & 0.5 \\
Uso dannoso di alcool (F10.1) & 5 & 2.0 & 2.6 \\
Episodio depressivo attuale (F32/33) & 34 & 13.6 & 4.7 \\
Distimia (F34) & 5 & 2.0 & 2.0 \\
Agorafobia (F40.0) & 5 & 2.0 & 0.6 \\
Sindrome da attacchi di panico (F41.0) & 4 & 1.6 & 1.5 \\
Sindrome d'ansia generalizzata (F41.1) & 21 & 8.4 & 3.7 \\
Sindrome da somatizzazione (F45.0) & 1 & 0.4 & 0.1 \\
Sindrome ipocondriaca (F45.2) & 1 & 0.4 & 0.3 \\
Nevrastenia (F48.0) & 16 & 6.4 & 2.1 \\
\hline Un disturbo psichico dei precedenti & 60 & 24.0 & 12.4 \\
Due o più disturbi psichici & 28 & 11.2 & 4.5 \\
\hline Totale & & & \\
\hline
\end{tabular}

guite nell'ordine dall'Uso Dannoso di Alcool $(2.6 \%)$, dalla Nevrastenia $(2.1 \%)$ e dalla Distimia $(2.0 \%)$; frequenze inferiori al $2 \%$ sono state invece riscontrate per gli altri disturbi indagati nel corso dello studio. Un ulteriore $11.2 \%$ dei soggetti selezionati presentava disturbi psichici 'sotto la soglia' (così definiti sulla base della presenza di sintomi in almeno due aree sintomatologiche distinte tra quelle previste dalle singole categorie diagnostiche dell'ICD-10, senza che venissero però soddisfatti tutti i criteri diagnostici per la presenza del disturbo in esame), mentre il $33.1 \%$ lamentava sintomi psicopatologici non inquadrabili in specifici criteri diagnostici o in un disturbo 'sotto la soglia' come sopra definito.

Come risulta dalle tabelle VII e VIII riportate alla fine, i disturbi psichici sono risultati più frequenti tra le donne rispetto agli uomini; lo stesso dicasi per la presenza contemporanea di due o più disturbi psichici diversi nello stesso soggetto. Anche per quanto riguarda le singole categorie diagnostiche considerate separatamente, la frequenza tra le femmine è risultata superiore rispetto a quella tra i maschi, fatta eccezione per i disturbi legati all'abuso di bevande alcooliche.

Nel loro complesso, i disturbi psichici sono risultati piuttosto rari tra i soggetti appartenenti alla classe di età più giovane $(3.9 \%)$ ed al contrario più fre- quenti tra coloro che avevano età compresa tra $25 \mathrm{e}$ 44 anni (14.0\%); viceversa, la presenza contemporanea di due o più disturbi psichici diversi è stata riscontrata soprattutto tra $\mathrm{i}$ soggetti di età compresa tra 45 e 65 anni $(5.5 \%)$.

\section{Motivo principale della consultazione}

La tabella IV mostra i principali motivi all'origine della consultazione con i servizi di medicina generale, quali sono stati riferiti dai pazienti nel corso della valutazione del secondo stadio.

Considerando l'intero campione (colonna a destra), circa la metà dei soggetti hanno riferito di aver consultato il medico per sintomi somatici (per esempio, disturbi dell'apparato respiratorio come la tosse, disturbi digestivi quali nausea, diarrea o stipsi, disturbi genito-urinari e così via) oppure per dolori in varie parti del corpo, mentre poco meno di un terzo dei soggetti si sono recati dal medico in occasione di visite periodiche legate a disturbi organici di lunga durata (vedi oltre) oppure per il rinnovo delle prescrizioni farmacologiche. Soltanto una ridotta percentuale delle consultazioni $(6.8 \%)$ è stata motivata da sintomi psicopatologici ben definiti, quali ansia o depressione; va inoltre considerata una piccola percentuale di pazienti $(3.3 \%)$ che si sono rivolti al medico per sintomi mal definiti per i quali si poteva sospettare la natura psicopatologica (disturbi del sonno, debolezza o sonnolenza, per esempio).

I motivi della consultazione sono stati indagati separatamente anche in tre gruppi distinti di soggetti: a) coloro che presentavano disturbi psichici ben definiti secondo l'ICD-10 (12.4\% del campione); b) quanti lamentavano disturbi organici cronici $(44.1 \%)$; c) coloro che non rientravano nelle due categorie precedenti $(\mathbf{4 3 . 5 \% )}$ ). Tra i disturbi organici cronici i più frequenti sono risultati l'ipertensione arteriosa $(19.5 \%$ del campione), l'artrite $(14.4 \%)$, la gastrite e/o l'ulcera peptica $(12.2 \%)$, i disturbi cardiaci $(5.1 \%)$, il diabete $(4.6 \%)$ e la bronchite e/o l'enfisema polmonare $(4.6 \%)$. È emerso che solamente il $20.6 \%$ dei soggetti con disturbi psichici si sono rivolti al medico per sintomi psicopatologici, il $5.7 \%$ lamentavano sintomi di possibile natura psicopatologica, mentre nel $70 \%$ circa dei casi il disturbo psichico è risultato mascherato da sintomi somatici, da dolori in varie parti del corpo oppure da disturbi organici cronici. Negli altri due gruppi di soggetti il medico è stato consultato essenzialmente per sintomi somatici, per dolori in varie parti del corpo oppure 
Tabella IV. - Motivo principale del contatto in rapporto con il disturbo presentato dal paziente.

\begin{tabular}{|c|c|c|c|c|c|c|c|c|}
\hline & \multicolumn{2}{|c|}{ Disturbi psichici } & \multicolumn{2}{|c|}{$\begin{array}{c}\text { Disturbi organici } \\
\text { cronici }\end{array}$} & \multicolumn{2}{|c|}{$\begin{array}{l}\text { Non disturbi psichici } \\
\text { né organici cronici }\end{array}$} & \multicolumn{2}{|c|}{ Campione totale } \\
\hline & $\mathbf{N}$ & $\% *$ & $\mathbf{N}$ & $\% *$ & $\mathbf{N}$ & $\% *$ & $\mathbf{N}$ & $\% *$ \\
\hline Sintomi psicopatologici & 18 & 20.6 & 9 & 5.6 & 10 & 4.2 & 37 & 6.8 \\
\hline Sintomi di possibile natura psicopatologica & 4 & 5.7 & 2 & 2.8 & 3 & 3.1 & 9 & 3.3 \\
\hline Dolori & 16 & 25.0 & 29 & 24.3 & 26 & 27.7 & 71 & 25.9 \\
\hline Sintomi somatici & 10 & 27.2 & 17 & 13.4 & 32 & 40.9 & 61 & 27.1 \\
\hline Assistenza pre- o post-natale & 1 & 1.0 & 2 & 1.0 & 4 & 5.7 & 7 & 3.0 \\
\hline Disturbi organici cronici & 7 & 16.5 & 21 & 29.3 & 0 & 0.0 & 26 & 15.0 \\
\hline Rinnovo prescrizione farmacologica & 2 & 2.0 & 14 & 18.5 & 17 & 17.6 & 33 & 16.1 \\
\hline Non conosciuto & 2 & 2.0 & 3 & 5.2 & 1 & 0.8 & 6 & 2.9 \\
\hline Totale & 60 & 100.0 & 97 & 100.0 & 93 & 100.0 & 250 & 100.0 \\
\hline
\end{tabular}

* Stime ponderate

per ragioni legate a disturbi organici cronici, mentre sintomi di certa o possibile natura psicopatologica erano all'origine della consultazione in poco meno del $10 \%$ dei casi.

\section{Stato di salute}

La tabella $\mathrm{V}$ riporta $\mathrm{i}$ risultati relativi al giudizio sullo stato complessivo di salute, espresso sia dai pazienti stessi (autovalutazione) sia dai medici di base partecipanti all'indagine.

Considerando l'intero campione (colonna a destra), poco più della metà dei soggetti hanno giudicato la propria salute come buona, molto buona o eccellente, mentre solo il 3.3\% l'hanno ritenuta francamente cattiva. La percezione da parte dei pazienti del proprio stato di salute è apparsa chiaramente influenzata dalla eventuale presenza di disturbi psichici o di disturbi organici cronici: infatti, il $62.0 \%$ dei soggetti con disturbi psichici hanno valutato la propria salute come discreta o cattiva, in confronto al $52.0 \%$ di quelli con disturbi organici cronici ed al $31.3 \%$ dei soggetti che non presentavano questo tipo di disturbi.

Secondo il giudizio espresso dai medici di base, nel complesso circa due terzi dei soggetti avevano uno stato di salute buono, molto buono o eccellente. Sempre secondo i medici, il $40.1 \%$ dei soggetti con disturbi psichici ed il $\mathbf{4 5 . 3 \%}$ di quelli con disturbi organici cronici godevano di salute discreta o cattiva contro il $14.4 \%$ di quelli che non presentavano tali disturbi.

\section{Livello di disabilità}

I risultati relativi ai livelli di disabilità, valutati in rapporto all'espletamento del proprio ruolo occupazionale e delle altre attività quotidiane, sono riportati nella tabella VI.

I risultati per l'intero campione (colonna a destra) indicano che due terzi circa dei soggetti non presentavano alcuna disabilità secondo il giudizio espresso tanto dal ricercatore quanto dai soggetti stessi (autovalutazione); peraltro il ricercatore ha mostrato la tendenza ad esprimere giudizi di minore gravità rispetto a quelli del paziente, quando identificava la presenza di disabilità.

La disabilità tendeva ad essere più frequente e più grave tra i soggetti con disturbi psichici rispetto a quelli con disturbi organici cronici o a coloro che non presentavano tali disturbi, pur con differenze tra il giudizio espresso dai pazienti e quello del ricercatore. Il $52.5 \%$ dei soggetti con disturbi psichici riteneva di avere qualche forma di disabilità e per il $40.1 \%$ questa era moderata o grave; tra $i$ soggetti con disturbi organici cronici, il $44.4 \%$ riteneva di presentare disabilità di vario grado e per il $26.8 \%$ questa era di livello moderato o grave; infine, solo il $15.0 \%$ dei soggetti senza questi disturbi riconoscevano qualche forma di disabilità e per il $9.1 \%$ questa era moderata o grave. Secondo il ricercatore, il $48.4 \%$ dei soggetti con disturbi psichici, il $39.0 \%$ di quelli con disturbi organici cronici, ed il $27.6 \%$ di quanti non avevano questi disturbi presentavano disabilità; questa era moderata o grave nel $14.8 \%$, $7.8 \%$ e $6.5 \%$ dei soggetti appartenenti ai tre gruppi, rispettivamente. 
M. Piccinelli, S. Pini, C. Bellantuono et al.

Tabella V. - Giudizio complessivo sullo stato di salute in rapporto con il disturbo presentato dal paziente.

\begin{tabular}{|c|c|c|c|c|c|c|c|c|}
\hline \multirow[t]{2}{*}{ Giudizio sulla salute } & \multicolumn{2}{|c|}{ Disturbi psichici } & \multicolumn{2}{|c|}{$\begin{array}{c}\text { Disturbi organici } \\
\text { cronici }\end{array}$} & \multicolumn{2}{|c|}{$\begin{array}{l}\text { non disturbi psichici } \\
\text { né organici cronici }\end{array}$} & \multicolumn{2}{|c|}{ Campione totale } \\
\hline & $\mathbf{N}$ & $\% *$ & $\mathbf{N}$ & $\% *$ & $\mathbf{N}$ & $\% *$ & $\mathbf{N}$ & $\% *$ \\
\hline \multicolumn{9}{|l|}{ Paziente } \\
\hline Eccellente & 1 & 1.0 & 0 & 0.0 & 6 & 6.6 & 7 & 3.0 \\
\hline Molto buona & 2 & 9.8 & 4 & 5.5 & 10 & 13.2 & 16 & 9.4 \\
\hline Buona & 9 & 26.3 & 40 & 41.7 & 45 & 41.3 & 94 & 39.6 \\
\hline Discreta & 36 & 50.0 & 49 & 50.7 & 27 & 28.5 & 112 & 40.9 \\
\hline Cattiva & 11 & 12.0 & 3 & 1.3 & 2 & 2.8 & 16 & 3.3 \\
\hline Non conosciuto & 0 & 0.0 & 1 & 0.7 & 1 & 2.5 & 5 & 3.7 \\
\hline \multicolumn{9}{|l|}{ Medico } \\
\hline Eccellente & 3 & 4.6 & 1 & 2.5 & 7 & 11.8 & 11 & 6.8 \\
\hline Molto buona & 4 & 3.9 & 10 & 8.1 & 21 & 21.3 & 35 & 13.3 \\
\hline Buona & 24 & 50.5 & 46 & 43.4 & 43 & 41.0 & 113 & 43.2 \\
\hline Discreta & 25 & 35.5 & 35 & 41.9 & 16 & 14.4 & 76 & 29.2 \\
\hline Cattiva & 3 & 4.6 & 3 & 3.4 & 0 & 0.0 & 6 & 2.1 \\
\hline Non conosciuto & 1 & 1.0 & 2 & 0.6 & 6 & 11.6 & 9 & 5.4 \\
\hline Totale & 60 & 100.0 & 97 & 100.0 & 96 & 100.0 & 250 & 100.0 \\
\hline
\end{tabular}

* Stime ponderate

Tabella VI. - Livelli di disabilità in rapporto con il disturbo presentato dal paziente.

\begin{tabular}{|c|c|c|c|c|c|c|c|c|}
\hline \multirow[t]{2}{*}{ Giudizio sulla salute } & \multicolumn{2}{|c|}{ Disturbi psichici } & \multicolumn{2}{|c|}{$\begin{array}{l}\text { Disturbi organici } \\
\text { cronici }\end{array}$} & \multicolumn{2}{|c|}{$\begin{array}{l}\text { non disturbi psichici } \\
\text { né organici cronici }\end{array}$} & \multicolumn{2}{|c|}{ Campione totale } \\
\hline & $\mathbf{N}$ & $\% *$ & $\mathbf{N}$ & $\%_{0}^{*}$ & $\mathbf{N}$ & $\% *$ & $\mathbf{N}$ & $\% *$ \\
\hline \multicolumn{9}{|l|}{ Giudizio paziente } \\
\hline Nessuna & 21 & 47.5 & 51 & 55.6 & 67 & 85.0 & 139 & 67.3 \\
\hline Lieve & 11 & 12.4 & 16 & 16.9 & 9 & 3,4 & 36 & 10.5 \\
\hline Moderata & 21 & 30.3 & 25 & 25.2 & 15 & 8.8 & 61 & 18.7 \\
\hline Grave & 7 & 9.8 & 4 & 1.6 & 1 & 0.3 & 12 & 2.0 \\
\hline Non conosciuto & 0 & 0.0 & 1 & 0.7 & 1 & 2.5 & 2 & 1.4 \\
\hline \multicolumn{9}{|l|}{ Giudizio ricercatore } \\
\hline Nessuna & 25 & 51.6 & 63 & 61.0 & 67 & 72.4 & 155 & 64.8 \\
\hline Lieve & 21 & 31.7 & 26 & 31.1 & 16 & 17.7 & 63 & 25.4 \\
\hline Moderata & 8 & 9.5 & 5 & 4.4 & 5 & 5.9 & 18 & 5.7 \\
\hline Grave & 4 & 5.3 & 3 & 3.4 & 2 & 0.6 & 9 & 2.4 \\
\hline Non conosciuto & 2 & 1.9 & 0 & 0.0 & 3 & 3.5 & 5 & 1.8 \\
\hline Totale & 60 & 100.0 & 97 & 100.0 & 93 & 100.0 & 250 & 100.0 \\
\hline
\end{tabular}

* Stime ponderate 
Presenza contemporanea di disturbi psichici e disturbi organici cronici

Il $60.0 \%$ dei soggetti con disturbi psichici secondo i criteri dell'ICD-10 lamentavano la presenza contemporanea di disturbi organici cronici. Questi soggetti esibivano un peggior stato complessivo di salute e più alti livelli di disabilità rispetto a quanti presentavano esclusivamente disturbi psichici: secondo il giudizio espresso dai pazienti, il $71.3 \%$ di quanti soffrivano contemporaneamente di disturbi psichici ed organici cronici dichiaravano di avere una salute discreta o cattiva rispetto al $49.4 \%$ di coloro con soli disturbi psichici; secondo il giudizio del medico di base, invece, nel $52.4 \%$ dei primi e nel $\mathbf{2 4 . 6 \%}$ dei secondi lo stato di salute era discreto o cattivo. Infine il $49.6 \%$ dei soggetti con disturbi psichici ed organici cronici ed il $27.1 \%$ di quelli con soli disturbi psichici lamentavano disabilità di grado moderato o grave; le percentuali corrispondenti secondo il giudizio del ricercatore erano $19.1 \%$ e $9.4 \%$ nei due gruppi, rispettivamente.

\section{DISCUSSIONE}

Sebbene in questo lavoro siano stati presentati unicamente alcuni dei risultati relativi all'area di Verona, occorre tuttavia ricordare che la presente indagine ha fatto parte di un progetto più ampio, in cui sono stati coinvolti altri 14 Centri, dei quali 7 situati in Europa, 3 in Asia e 2 in America Latina, mentre Africa e Stati Uniti potevano contare su un Centro ciascuno. In tal modo, è stato possibile raccogliere una mole notevole di dati in grado di rispecchiare realtà socio-economiche e culturali tra loro molto diverse ed è stata assicurata la creazione di un programma di attiva collaborazione tra ricercatori di varia formazione impegnati nel campo della salute mentale, con una conseguente ampia diffusione di conoscenze.

\section{Frequenza dei disturbi psichici}

Tra i risultati ottenuti a Verona colpisce la frequenza relativamente bassa di disturbi psichici secondo i criteri diagnostici dell'ICD-10. Tale frequenza $(12.4 \%)$ è risultata, infatti, inferiore rispetto a quella riportata da precedenti studi condotti in Ita- lia: in Emilia-Romagna, Berti Ceroni et al. (1992) hanno trovato che il $22.1 \%$ dei pazienti selezionati negli ambulatori di medicina generale soddisfacevano i criteri diagnostici del DSM-III (American Psychiatric Association, 1980) per la presenza di disturbi psichici; nell'area di Verona, invece, Marino et al. (1990) hanno stimato la frequenza di disturbi psichici intorno al $40 \%$, quando il General Health Questionnaire è stato fatto completare ai soggetti $(\mathrm{N}=404)$ che si erano rivolti a 32 ambulatori di medicina generale nel corso di un singolo giorno.

Nel tentativo di tenere conto del possibile effetto confondente legato all'impiego di strumenti e criteri diagnostici differenti, la frequenza dei disturbi psichici nel nostro campione è stata ricalcolata impiegando tanto i criteri diagnostici del DSM-III-R (American Psychiatric Association, 1987) quanto i punteggi ottenuti al GHQ-12 durante la procedura di screening. Facendo riferimento ai criteri del DSMIII-R, la frequenza di disturbi psichici nel nostro campione è risultata pari a $10.1 \%$, la metà circa di quella riscontrata da Berti Ceroni et al. (1992). A parte le diverse versioni del DSM impiegate, occorre tuttavia considerare che le due frequenze non sono direttamente confrontabili, essendo basate su categorie diagnostiche diverse: così nella nostra indagine sono stati esclusi i disturbi dell'adattamento e quelli di personalità, che figuravano invece nello studio di Berti Ceroni et al. (1992); al contrario, in quest'ultimo non sono stati inclusi i disturbi legati all'abuso di alcool, che nella nostra indagine sono risultati $i$ disturbi più frequenti tra gli uomini. D'altra parte, poiché Berti Ceroni et al. (1992) non hanno riportato le frequenze distinte per singole categorie diagnostiche, non è stato possibile apportare alcuna correzione al valore della frequenza totale al fine di assicurare la confrontabilità dei risultati.

Seguendo il metodo proposto da Goldberg (1972) e basato su di un semplice sviluppo del teorema di Bayes, la frequenza di disturbi psichici nel nostro campione è stata calcolata anche sulla base dei punteggi riportati al GHQ-12 e dei coefficienti di validità del questionario ottenuti nel corso della sua validazione nel contesto della medicina generale (Piccinelli et al., 1993). La frequenza stimata di disturbi psichici è risultata così pari a $23.1 \%$, più elevata rispetto a quella basata sui criteri diagnostici dell'ICD-10, ma pur sempre inferiore rispetto a quella ottenuta in maniera analoga da Marino et al. (1990). Due ipotesi possono essere avanzate nel tentativo di spiegare tale differenza. Innanzitutto, non è possibile escludere che il diverso disegno dei due studi abbia 
influito sui risultati: poiché è stato ripetutamente dimostrato che i pazienti con disturbi psichici tendono a rivolgersi ai servizi di medicina generale più frequentemente rispetto a coloro che non hanno questi disturbi (Finlay-Jones \& Burvill, 1978; Williams et al., 1986; Vazquez-Barquero et al., 1990), il fatto che Marino et al. (1990) abbiano scelto un singolo giorno durante il quale operare la selezione dei pazienti può aver contribuito ad innalzare la stima di frequenza dei disturbi psichici, essendo maggiore la probabilità di selezionare soggetti con questo tipo di disturbi. Al contrario, nella nostra indagine la frequenza dei disturbi psichici ottenuta può essere ritenuta relativamente indipendente dalle caratteristiche di consultazione dei servizi, considerato sia il lungo periodo di tempo durante il quale si è svolta la selezione sia il fatto che ogni paziente ha fornito dati una volta soltanto durante il periodo dello studio; inoltre, nel corso della nostra indagine, per ogni medico si è cercato, per quanto possibile, di far ruotare le sessioni ambulatoriali durante le quali è stata condotta la procedura di screening al fine di evitare eventuali fenomeni di auto-selezione dei pazienti.

La seconda ipotesi si riferisce, invece, alla possibilità che la frequenza dei disturbi psichici tra gli utenti dei servizi di medicina generale nell'area di Verona si sia effettivamente ridotta nel tempo, a causa della diminuzione delia prevalenza dei disturbi psichici nella popolazione generale oppure del cambiamento delle modalità di trattamento scelte dai soggetti con questo tipo di disturbi (per esempio, aumento della quota di pazienti con disturbi psichici che si rivolgono direttamente al servizio psichiatrico pubblico oppure a psichiatri e psicologi privati, senza prima contattare il medico di base) oppure di entrambi questi fattori. Sfortunatamente non abbiamo a disposizione dati per verificare eventuali cambiamenti intervenuti nella prevalenza dei disturbi psichici nell'area di Verona tra il 1987, anno in cui è stato condotto lo studio di Marino et al. (1990), e gli anni 1991-1992, nel corso dei quali si è svolta la nostra indagine; lo stesso dicasi per quanto riguarda l'assistenza fornita a livello privato a soggetti con disturbi psichici. Utilizzando, invece, i dati forniti dal Registro Psichiatrico dei Casi di Verona-Sud sono stati esaminati i committenti dei primi contatti in assoluto (first-ever) con il servizio psichiatrico territoriale relativi agli anni 1987 e 1991. Nel 1987, 255 soggetti sono entrati in contatto per la prima volta con il servizio psichiatrico, il $23.9 \%$ essendovi inviati dal medico di base, il $12.1 \%$ da altri medici (soprattutto specialisti privati od ospedalieri) ed il $55.7 \%$ essen- dovi giunti direttamente in seguito alla richiesta del paziente stesso o dei suoi familiari; nel 1991, i soggetti first-ever per il servizio sono stati 201 e di questi il $22.9 \%$ sono stati inviati dal medico di base, il $21.9 \%$ da altri medici, mentre nel $42.8 \%$ dei casi il contatto è stato cercato dal paziente stesso o dai suoi familiari. È evidente che questi dati forniscono un'immagine qual'è vista dal versante del servizio psichiatrico, mentre nulla ci dicono circa i percorsi assistenziali seguiti dai pazienti, fatta eccezione per l'ultimo committente del contatto in ordine di tempo. Pur con questi limiti, i dati disponibili sembrano suggerire una diminuzione del numero annuale di primi contatti con il servizio psichiatrico; inoltre, se il ruolo del medico di base nell'invio di pazienti al servizio è rimasto proporzionalmente stabile, quello degli altri medici è invece aumentato. Purtroppo non ci è dato sapere quale quota di pazienti con disturbi psichici ha finito per ricevere trattamento esclusivamente da questi ultimi, senza avere contatti né con i servizi di medicina generale da una parte né con quelli psichiatrici dall'altra.

Volendo collocare la frequenza dei disturbi psichici riscontrata a Verona in una prospettiva internazionale, con riferimento cioè agli altri Centri partecipanti allo studio descritto in questo lavoro, è interessante rilevare come essa sia risultata superiore solamente alle frequenze riportate a Shangai $(9.8 \%)$ e ad Ibadan $(10.4 \%)$, ma inferiore rispetto a quelle trovate negli altri sette Centri europei (mediana 26.3\%; intervallo compreso tra $17.6 \%$ e $31.3 \%$ ); analogamente, essa si colloca al limite inferiore della distribuzione delle frequenze dei disturbi psichici riportate da altri studi condotti nella medicina generale secondo procedure standardizzate (per una revisione sull'argomento, si rimanda a Piccinelli, 1994).

Non è facile comprendere le ragioni di queste differenze. Accanto alla diversa suscettibilità nei confronti dei disturbi psichici potenzialmente presente in Paesi diversi (in rapporto, per esempio, all'ineguale distribuzione di fattori di rischio di tipo economico, sociale o culturale), occorre considerare altre possibilità. Sebbene, infatti, particolare attenzione sia stata rivolta alla standardizzazione degli strumenti e delle procedure d'indagine impiegati in tutti i Centri, è evidente che queste precauzioni non sono sempre sufficienti al fine di superare possibili differenze culturali relative all'attitudine ed alla disponibilità dei pazienti a rivelare i propri problemi psicologici tanto al medico di base quanto ai ricercatori, verosimilmente in rapporto al diverso modo in cui i disturbi psichici sono compresi e concettualizzati in Paesi dif- 
ferenti ed al maggiore o minore stigma sociale ad essi legato. Inoltre, le caratteristiche dell'assistenza sanitaria variano spesso in misura considerevole da Paese a Paese e fattori quali la disponibilità e l'organizzazione dei servizi di medicina generale, la facilità di accesso ad essi, la presenza di strutture alternative di trattamento o di assistenza specialistica, la possibilità di ricevere vari tipi di trattamento, il costo necessario per usufruire delle prestazioni sanitarie rappresentano altrettanti elementi in grado di influire sulle stime di frequenza, ma difficilmente controllabili nel corso di confronti trans-culturali.

Infine, un commento a parte meritano i disturbi psichici cosiddetti 'sotto la soglia'. A questo proposito, è importante sottolineare che un disturbo psichico può rivestire rilevanza clinica nonostante non soddisfi tutti i criteri di uno dei maggiori sistemi di classificazione e di ciò va tenuto conto sul piano dell'intervento terapeutico. Klerman (1989) ha osservato, infatti, che soggetti con sintomi depressivi non inquadrabili secondo il DSM-III nelle categorie diagnostiche Depressione Maggiore e/o Distimia presentavano maggiori limitazioni in numerose aree del funzionamento sociale se confrontati con controlli normali e con pazienti affetti da condizioni organiche croniche. Inoltre, Wells et al. (1989) hanno rilevato che gli utenti dei servizi di medicina generale che lamentavana sintomi depressivi ma non soddisfacevano i criteri del DSM-III per un disturbo depressivo ben definito presentavano limitazioni nel funzionamento fisico, sociale ed occupazionale e nel benessere soggettivo sovrapponibili a quelle sofferte dai soggetti con disturbi depressivi ben definiti secondo il DSM-III stesso. Infine, Johnson et al. (1992) hanno utilizzato i dati raccolti negli Stati Uniti durante l'Epidemiologic Catchment Area Study per stimare l'utilizzazione dei servizi sanitari, il consumo di farmaci psicotropi, la perdita di giornate lavorative, il numero di tentativi di suicidio ed il livello di salute associati alla presenza di sintomi depressivi non inquadrabili nelle categorie diagnostiche Depressione Maggiore e/o Distimia secondo il DSMIII. In generale, i soggetti che soddisfacevano i criteri diagnostici per la Depressione Maggiore e/o la Distimia avevano un rischio relativo più elevato per ciascuna delle variabili considerate rispetto a quanti presentavano esclusivamente sintomi depressivi. D'altra parte, i soggetti che lamentavano sintomi depressivi in assenza di un disturbo depressivo ben definito erano responsabili nel loro insieme di una maggiore utilizzazione complessiva dei servizi sanitari e presentavano maggiori limitazioni sul piano so- ciale rispetto a quanti soffrivano di disturbi depressivi ben definiti, in virtù della loro prevalenza più elevata nella popolazione. Ne deriva che i disturbi psichici cosiddetti 'sotto la soglia', pur non soddisfacendo tutti i criteri contenuti negli attuali sistemi nosografici dei disturbi psichici, rappresentano un importante problema clinico e di salute pubblica e, come tali, meritano un'attenzione speciale sul piano classificatorio e del trattamento.

\section{Co-presenza di disturbi psichici}

e disturbi organici cronici

L'elevata frequenza con cui nel nostro campione i soggetti con disturbi psichici hanno lamentato la contemporanea presenza di disturbi organici cronici di vario tipo rappresenta un'osservazione frequentemente riportata in letteratura. Nel classico studio condotto da Shepherd e collaboratori (Sheperd et al., 1966; Sheperd \& Clare, 1981) in 46 ambulatori di medicina generale nell'area di Londra, i tassi di disturbi organici cronici risultavano più elevati tra $i$ pazienti con disturbi psichici rispetto a quanti non avevano questi disturbi, fatta eccezione per le malattie neoplastiche in entrambi i sessi e quelle ortopediche nelle donne; quando poi i disturbi organici cronici e quelli acuti sono stati considerati insieme, è risultato che i soggetti con disturbi psichici presentavano un numero medio di disturbi organici significativamente superiore rispetto agli altri pazienti ed avevano consultato più frequentemente i servizi di medicina generale per entrambi i tipi di disturbi, non solo cioè di natura psichica ma anche di tipo organico.

Analogamente, Wilkinson et al. (1988), analizzando i contatti per disturbi organici avuti dai pazienti iscritti nella lista di un singolo medico di base nell'arco di un periodo di 20 anni, hanno riscontrato che contatti di questo tipo risultavano più frequenti tra i pazienti con disturbi psichici, interessando circa il $90 \%$ dei soggetti con disturbi psichici che avevano ricevuto una nuova prescrizione di farmaci psicotropi (definita tale quando il farmaco non era stato prescritto nei precedenti sei mesi), $1^{\prime} 85 \%$ di quelli con disturbi psichici ma senza una nuova prescrizione di farmaci psicotropi, ed il $60 \%$ dei soggetti di controllo.

Infine, più recentemente negli Stati Uniti, Wells et al. (1991) hanno indagato la co-occorrenza di disturbi organici in 1.152 soggetti che presentavano sintomi depressivi al momento della selezione nei servizi di medicina generale e di salute mentale e 
soddisfacevano i criteri del DSM-III per la diagnosi lifetime di disturbo depressivo unipolare. È emerso così che il $71.0 \%$ dei pazienti selezionati nei servizi di medicina generale ed il $64.9 \%$ di quelli che si erano rivolti ai servizi di salute mentale presentavano almeno un disturbo organico cronico concomitante. I disturbi organici più frequenti tra i pazienti dei servizi di medicina generale sono risultati l'ipertensione arteriosa $(43.1 \%)$, l'artrite $(26.2 \%)$, i disturbi respiratori cronici $(18.8 \%)$, il diabete $(13.4 \%)$ ed i disturbi gastro-intestinali (11.4\%); inoltre, i soggetti che avevano consultato i servizi di medicina generale lamentavano ipertensione arteriosa ed artrite con frequenza significativamente maggiore rispetto a quelli visti nei servizi di salute mentale (rispettivamente, $43.1 \%$ contro $24.4 \%$ e $26.2 \%$ contro $20.3 \%$ ) ed avevano maggiore probabilità di assumere un trattamento anti-ipertensivo o insulinico.

\section{Motivo principale della consultazione}

Nel nostro campione, disturbi organici cronici, sintomi somatici e dolori in varie parti del corpo figuravano come la ragione addotta dal $70 \%$ circa dei soggetti con disturbi psichici per aver consultato i servizi di medicina generale. Questi risultati confermano come spesso nel contesto della medicina generale i disturbi psichici non vengano portati come tali alla diretta attenzione del medico, ma rimangano celati dietro la presentazione di una sintomatologia di natura somatica. Si tratta di un fenomeno ben conosciuto che è stato studiato secondo due prospettive diverse, ma complementari: da un lato, è stata indagata la frequenza con cui soggetti con disturbi psichici ben definiti secondo gli attuali sistemi nosografici rivelano al medico unicamente sintomi somatici invece di quelli psicopatologici; dall'altro, è stata analizzata la frequenza con cui soggetti che lamentano sintomi somatici non spiegabili su base organica soddisfano i criteri diagnostici per un disturbo psichico ben definito (diverso dai disturbi somatoformi).

In riferimento al primo dei due approcci menzionati, risultati interessanti sono stati riportati da un'indagine condotta in 15 ambulatori di medicina generale nell'area di Manchester, dalla quale è emerso che un disturbo psichico secondo i criteri del DSM-III era presente nel $33 \%$ dei pazienti che iniziavano un nuovo episodio di cura (definito come tale in quanto non erano stati consultati altri medici nei 12 mesi precedenti l'indagine). Peraltro, solo il
$17 \%$ di questi pazienti avevano comunicato al medico il proprio disagio in termini psicologici, il $27 \%$ avevano consultato il medico per un disturbo organico concomitante, mentre il $56 \%$ avevano lamentato esclusivamente sintomi somatici e ritenevano tali sintomi indicativi di un disturbo organico, a differenza dello psichiatra secondo il quale il trattamento del disturbo psichico avrebbe ridotto o fatto scomparire i sintomi somatici (Bridges \& Goldberg, 1985).

Il rapporto intercorrente tra disturbi psichici e sintomi somatici inspiegabili su base strettamente organica è stato indagato da diversi studi, i quali hanno sottolineato l'elevata frequenza con cui disturbi psichici ben definiti possono essere diagnosticati nei pazienti che riportano questo tipo di sintomi. Utilizzando i dati relativi alla popolazione generale raccolti negli Stati Uniti nel corso dell'Epidemiologic Catchment Area Study, Simon \& Von Korff (1991) hanno trovato che, tra i soggetti che lamentavano cinque $o$ più sintomi somatici cosiddetti funzionali, il $63.7 \%$ riportavano anche uno o più sintomi psicopatologici (periodi di depressione, episodi di pianto, attacchi di panico e così via) ed il $49.2 \%$ avevano un vero e proprio disturbo depressivo o ansioso; al contrario, tra quanti non avevano sintomi somatici funzionali solo il $7.1 \%$ lamentavano sintomi psicopatologici ed il $5.5 \%$ presentavano un disturbo depressivo o ansioso. Una correlazione lineare tra numero di sintomi somatici senza spiegazione medica e disturbi depressivi ed ansiosi è stata riportata anche da Katon et al. (1991) in un campione di pazienti della medicina generale. Ancora, in uno studio condotto in un reparto di medicina ospedaliero in Olanda è emerso che il $45 \%$ dei pazienti ricoverati cui era stata assegnata una diagnosi medica dubbia presentavano disturbi psichici (soprattutto, di tipo depressivo ed ansioso) ad un'intervista psichiatrica in confronto al $38 \%$ di quelli che non avevano ricevuta alcuna diagnosi medica ed al $15 \%$ di quelli con diagnosi mediche ben definite (Van Hemert et al., 1993). Infine, elevata frequenza di disturbi psichici, soprattutto di tipo depressivo, è stata dimostrata non solo tra $i$ soggetti definiti genericamente come 'somatizzatori' (Katon et al., 1984), ma anche in pazienti con diversi tipi di disturbi tuttora mal definiti sul piano ezio-patogenetico, quali dolore cronico (Roy et al., 1984; Walker et al., 1988), dolore toracico atipico (Cormier et al., 1988), tinnito (Sullivan et al., 1988) e sindrome da fatica cronica (Kruesi et al., 1989).

Quando Bridges et al. (1991) hanno confrontato i soggetti che esprimevano il loro disturbo psichico sotto forma di sintomi somatici (definiti 'somatizza- 
tori') con quelli che invece riferivano sintomi psicopatologici ('psicologizzatori') non sono emerse differenze significative tra $\mathrm{i}$ due gruppi per quanto riguarda caratteristiche socio-demografiche, livello d'intelligenza, esperienze infantili, caratteristiche familiari attuali e rapporti con il coniuge, tratti di personalità come ipocondria, desiderabilità sociale o difensività. Tuttavia, i somatizzatori rivelavano un'attitudine meno positiva nei confronti delle malattie mentali, erano meno inclini a rivelare sintomi psicopatologici al loro medico ed avevano avuto più ricoveri in reparti medici ospedalieri; inoltre, essi presentavano un più basso livello di disagio psicologico, espresso dal punteggio totale alla Present State Examination (Wing \& Sturt, 1978) ed al General Health Questionnaire, ed erano meno depressi secondo il giudizio clinico.

Viene in tal modo avvalorata l'ipotesi che il processo di somatizzazione abbia una funzione adattati$\mathrm{va}$, in quanto consentirebbe di assumere il ruolo di malato senza sentirsi direttamente responsabili della propria condizione e scegliendo una malattia socialmente più rispettabile rispetto ad un disturbo psichico (Goldberg \& Bridges, 1988). Questo possibile vantaggio è tuttavia controbilanciato dal fatto che i pazienti che somatizzano il proprio disagio psichico tendono ad avere disturbi che durano più a lungo, consultano più frequentemente i servizi sanitari ed assumono più spesso farmaci, soprattutto analgesici (Huxley \& Goldberg, 1975; Katon et al., 1984; Escobar et al., 1987).

Queste osservazioni sottolineano, dunque, la necessità e l'importanza di migliorare la capacità del medico di base di riconoscere prontamente questi disturbi tramite appropriati programmi di training, considerate le difficoltà diagnostiche poste da questi pazienti. Per esempio, nello studio di Bridges \& Goldberg (1985) i medici di base avevano diagnosticato la presenza di un disturbo psichico nel $\mathbf{9 0 \%}$ dei pazienti che presentavano il loro disturbo sotto forma di sintomi psicopatologici, nel $73 \%$ di coloro nei quali il disturbo psichico era secondario ad una malattia organica accertata, ma solo nel $50 \%$ dei pazienti che avevano adottato il processo di somatizzazione. Contemporaneamente, è opportuno sviluppare e diffondere semplici strategie di trattamento basate sulla 'riattribuzione' dei sintomi somatici non più ad una causa organica, ma ad una psicologica, considerati i promettenti risultati riportati in letteratura (Smith et al., 1986; Barsky et al., 1988; Warwick, 1989; Gask , 1992).
Stato di salute e livello di disabilità

La percezione soggettiva del proprio stato di salute da parte del paziente è apparsa influenzata dalla eventuale presenza di disturbi psichici o di disturbi organici cronici, dal momento che il $60 \%$ circa dei soggetti con disturbi psichici ed il $50 \%$ circa di quelli con disturbi organici cronici hanno valutato la propria salute come discreta o cattiva in confronto al $30 \%$ circa dei soggetti che non presentavano questo tipo di disturbi. Una tendenza simile è stata riscontrata anche negli Stati Uniti analizzando i dati raccolti nel corso dell'Epidemiologic Catchment Area Study. In questo studio, è stato dimostrato che la percentuale di soggetti che consideravano la propria salute come discreta o cattiva era superiore tra quanti presentavano disturbi psichici e/o organici cronici rispetto a coloro che non avevano questo tipo di disturbi: in particolare, tale percentuale è risultata pari a $44.1 \%$ tra i soggetti che avevano contemporaneamente disturbi psichici ed organici cronici, $33.3 \%$ tra quanti soffrivano unicamente di disturbi organici cronici, $20.7 \%$ tra coloro che avevano solo disturbi psichici e $12.5 \%$ tra quanti non presentavano disturbi di questo tipo (Wells et al., 1988). Rispetto ai risultati riportati da questi autori una percentuale superiore di soggetti inclusi nella nostra indagine hanno fornito una valutazione negativa del proprio stato di salute. Questo può spiegarsi col fatto che il nostro campione è stato selezionato tra coloro che si erano rivolti ai servizi di medicina generale e non nella popolazione generale, per cui possono sussistere differenze tra i due campioni per quanto riguarda la durata e/o la gravità dei disturbi organici e psichici riportati dai pazienti.

L'autovalutazione dello stato di salute è considerata una misura complessiva del modo in cui vari aspetti che concorrono alla definizione di salute sono percepiti dai singoli individui. Un simile giudizio sembra basarsi su una valutazione più ampia e generale rispetto a quella adottata in conformità ad una visione puramente medica: mentre, infatti, il medico tende ad adottare un criterio di esclusione nel considerare un individuo in buona salute (avendo a sua disposizione una serie di esami e test diagnostici volti appunto ad escludere la presenza di una malattia), il paziente, invece, associa il concetto di salute all'apparente buon funzionamento del proprio organismo e in genere non fa distinzione tra salute fisica e salute mentale, come se queste fossero entità separate, né prescinde dalla sua capacità di rispondere o meno alle richieste dell'ambiente in cui si trova, coinvol- 
gendo in tal modo nella sua valutazione anche il contesto materiale e sociale in cui vive. In effetti, in uno studio condotto in Norvegia volto ad indagare i meccanismi implicati nella valutazione soggettiva dello stato di salute attraverso l'esame dei rapporti intercorrenti tra stato di salute fisica e psicologica, caratteristiche comportamentali e fattori sociali, è stato dimostrato che il disagio fisico (misurato in riferimento a sintomi di dolore in varie parti del corpo ed inteso dagli autori come un indice piuttosto generale, essendo influenzato non solo da malattie e disturbi fisici, ma anche dalle condizioni psicologiche individuali e dall'occorrenza di eventi stressanti ambientali) e la fruizione di un contributo per invalidità lavorativa permanente erano $i$ fattori più importanti nell'abbassare la valutazione relativa alla propria salute, mentre l'effetto delle malattie organiche croniche e del disagio psicologico sul giudizio di salute si esplicavano in via indiretta attraverso i due fattori precedenti (Fylkesnes \& Forde, 1991; 1992).

A parziale conferma delle differenze di giudizio che possono sussistere tra i pazienti ed il proprio medico nel valutare lo stato di salute, è possibile notare come $\mathrm{i}$ medici coinvolti nella nostra indagine abbiano assegnato in genere giudizi più positivi rispetto a quelli dei pazienti ed abbiano valorizzato soprattutto la presenza di disturbi organici cronici nell'assegnare una valutazione di compromissione dello stato di salute. Secondo il giudizio dei medici, infatti, il $45 \%$ circa dei soggetti con disturbi organici cronici godevano di salute discreta o cattiva rispetto al $40 \%$ circa dei soggetti con disturbi psichici ed al $14 \%$ di quelli che non presentavano tali disturbi; considerando poi esclusivamente i soggetti con disturbi psichici, solo il $25 \%$ di quanti non presentavano disturbi organici cronici concomitanti ma il $52 \%$ di coloro con tali disturbi hanno ricevuto un simile giudizio dal medico, a differenza delle valutazioni espresse dai pazienti, secondo le quali un giudizio di salute discreta o cattiva interessava rispettivamente il $71 \%$ ed il $49 \%$ dei soggetti con e senza disturbi organici cronici concomitanti.

Nella nostra indagine i disturbi psichici si sono rivelati invalidanti per $i$ soggetti che ne erano portatori: la disabilità occupazionale e nelle altre attività quotidiane tendeva, infatti, ad essere più frequente $\mathrm{e}$ più grave tra i soggetti con disturbi psichici rispetto a quelli con disturbi organici cronici o coloro che non presentavano tali disturbi. Risultati simili sono stati riportati da Wells et al. (1989) confrontando lo stato di disabilità dei soggetti affetti da un disturbo depressivo con quello dei soggetti con e senza condi- zioni organiche croniche (tra queste ultime figuravano: storia di ipertensione; storia di diabete; malattia coronarica in fase avanzata; angina; artrite; problemi alla schiena; problemi polmonari; disturbi gastrointestinali). In generale, il livello di disabilità è risultato superiore nei pazienti depressi rispetto a quelli senza condizioni organiche croniche; inoltre, nei pazienti depressi il funzionamento sociale è risultato più basso rispetto a quello dei soggetti con una delle condizioni organiche croniche elencate; lo stesso dicasi per il ruolo occupazionale (con l'eccezione dei soggetti con malattia coronarica in fase avanzata) ed il funzionamento fisico (con l'eccezione dei soggetti con malattia coronarica in fase avanzata, angina, problemi alla schiena, problemi polmonari). Anche i giorni trascorsi a letto nell'ultimo mese sono risultati superiori nei pazienti depressi rispetto a quelli con condizioni organiche croniche (con l'eccezione dei soggetti con malattia coronarica in fase avanzata e problemi polmonari).

Più recentemente, Wohlfarth et al. (1993) hanno valutato il livello di disabilità sociale tra i pazienti della medicina generale in Olanda, utilizzando lo stesso strumento impiegato nella nostra indagine (Social Disability Schedule), ma esplorando anche altre aree oltre al ruolo occupazionale ed alle attività quotidiane. Peraltro, dati completi erano disponibili solo per i pazienti selezionati che non avevano ricevuto dal medico di base una diagnosi di disturbo psichico nei dodici mesi precedenti l'indagine, per cui non è possibile un confronto diretto con $\mathrm{i}$ risultati ottenuti nel nostro campione. La disabilità, in gran parte limitata al ruolo occupazionale e a quello sociale, è risultata piuttosto limitata, ma più pronunciata nei soggetti con disturbi psichici rispetto a quelli senza tali disturbi; inoltre, la disabilità occupazionale e sociale e quella totale erano moderatamente correlate con la gravità del disturbo psichico, espresso dall'indice di definizione alla Present State Examination, e con la diagnosi (più elevate nei soggetti depressi). Quando il campione di pazienti con disturbi psichici alla valutazione iniziale è stato riesaminato a distanza di un anno e poi di 3.5 anni, l'andamento dei livelli di disabilità è risultato in rapporto con quello del quadro psicopatologico; inoltre, nonostante il miglioramento statisticamente significativo intervenuto nel corso del periodo di follow-up in tutte le categorie diagnostiche, è rimasta un'apprezzabile disabilità residua soprattutto nel ruolo sociale e tra i pazienti con co-occorrenza di disturbi d'ansia e depressivi (Ormel et al., 1993).

Questi risultati suggeriscono che i disturbi psichici 
che si riscontrano nel contesto della medicina generale possono causare serie limitazioni e marcata sofferenza per i pazienti e comportare costi elevati per la società. In effetti, i costi sostenuti nel 1985 in Gran Bretagna per i disturbi nevrotici trattati esclusivamente nei servizi di medicina generale sono risultati simili a quelli richiesti da un altro disturbo molto frequente tra i pazienti del medico di base, cioè l'ipertensione arteriosa, ed ammontavano a circa il $9 \%$ delle spese complessive sostenute in quell'anno dal governo inglese per i servizi di medicina generale. Tuttavia, mentre per i pazienti ipertesi gran parte dei costi erano imputabili ai farmaci impiegati per il trattamento, per i disturbi nevrotici si trattava soprattutto di costi indiretti legati alla perdita di produttività in campo lavorativo (Croft-Jeffreys \& Wilkinson, 1989).

\section{CONCLUSIONI}

In sintesi, i risultati ottenuti nel corso della nostra indagine si prestano alle seguenti considerazioni:

(I) Disturbi psichici ben definiti secondo i criteri dell'ICD-10 erano presenti in un paziente su otto $(12.4 \%)$ tra quanti contattavano i servizi di medicina generale; considerando anche i disturbi psichici 'sotto la soglia', disturbi psichici erano presenti all'incirca in un paziente su quattro (23.6\%); infine, sintomi psicopatologici, indipendentemente da qualsivoglia criterio nosografico, sono stati lamentati da più della metà dei soggetti nel nostro campione $(56.6 \%)$;

(II) I disturbi psichici sono risultati più frequenti nel sesso femminile. Tra i più comuni vanno annoverati quelli affettivi (Episodio Depressivo e Distimia) e quelli ansiosi (Sindrome d'Ansia Generalizzata); nel sesso maschile sono risultati frequenti i disturbi legati all'abuso di bevande alcooliche;

(III) La maggior parte dei soggetti con disturbi psichici ben definiti secondo i criteri dell'ICD-10 non hanno presentato al medico sintomi psicopatologici, ma piuttosto una sintomatologia di natura somatica; inoltre, più della metà di questi soggetti lamentavano almeno un disturbo organico cronico concomitante. Queste osservazioni sottolineano le difficoltà che il medico di base deve affrontare sul piano del riconoscimento e del trattamento dei disturbi psichici presenti nella sua pratica clinica quotidiana;

(IV) Più della metà dei soggetti con disturbi psichici hanno riportato un giudizio negativo sul proprio stato di salute e limitazioni di vario grado in
Tabella VII. - Distribuzione dei disturbi psichici attuali secondo l'ICD-10 per sesso.

\begin{tabular}{|c|c|c|c|c|}
\hline & \multicolumn{2}{|c|}{ Maschi } & \multicolumn{2}{|c|}{ Femmine } \\
\hline & $\mathbf{N}$ & $\% *$ & $\mathbf{N}$ & $\% *$ \\
\hline Dipendenza da alcool (F10.2) & 2 & 1.1 & 1 & 0.2 \\
\hline Uso dannoso di alcool (F10.17 & 5 & 7.1 & 0 & 0.0 \\
\hline Episodio depressivo attuale (F32/33) & 8 & 3.2 & 26 & 5.5 \\
\hline Distimia (F34) & 0 & 0.0 & 5 & 3.1 \\
\hline Agorafobia (F40.0) & 0 & 0.0 & 5 & 0.9 \\
\hline Sindrome da attacchi di panico (F41.0) & 1 & 0.4 & 3 & 2.1 \\
\hline Sindrome d'ansia generalizzata (F41.1) & 3 & 1.5 & 18 & 4.9 \\
\hline Sindrome da somatizzazione (F45.0) & 0 & 0.0 & 1 & 0.2 \\
\hline Sindrome ipocondriaca (F45.2) & 0 & 0.0 & 1 & 0.2 \\
\hline Nevrastenia (F48.0) & 4 & 1.8 & 12 & 2.3 \\
\hline Un disturbo psichico & 14 & 10.6 & 46 & 13.4 \\
\hline Due o più disturbi psichici & 6 & 2.5 & 22 & 5.7 \\
\hline Totale & 83 & 100.0 & 167 & 100.0 \\
\hline
\end{tabular}

* Stime ponderate

Tabella VIII. - Distribuzione dei disturbi psichici attuali secondo l'ICD-10 per classi d'età.

\begin{tabular}{|c|c|c|c|c|c|c|}
\hline \multirow{3}{*}{ Categoria diagnostica } & \multicolumn{6}{|c|}{ Età } \\
\hline & \multicolumn{2}{|c|}{$18-24$} & \multicolumn{2}{|c|}{$25-44$} & \multicolumn{2}{|c|}{$45-65$} \\
\hline & $\mathbf{N}$ & $\% *$ & $\mathbf{N}$ & $\% 0^{*}$ & $\mathbf{N}$ & $\% *$ \\
\hline Dipendenza da alcool (F10.2) & 0 & 0.0 & 3 & 1.3 & 0 & 0.0 \\
\hline Uso dannoso di alcool (F10.1) & 0 & 0.0 & 3 & 5.6 & 2 & 0.5 \\
\hline \multicolumn{7}{|l|}{ Episodio depressivo attuale } \\
\hline$(\mathrm{F} 32 / 33)$ & 0 & 0.0 & 19 & 6.0 & 15 & 4.8 \\
\hline Distimia (F34) & 0 & 0.0 & 1 & 0.8 & 4 & 3.6 \\
\hline Agorafobia (F40.0) & 0 & 0.0 & 4 & 1.2 & 1 & 0.3 \\
\hline \multicolumn{7}{|l|}{ Sindrome da attacchi di panico } \\
\hline$(\mathrm{F} 41.0)$ & 1 & 1.0 & 2 & 0.6 & 2 & 2.6 \\
\hline Sidrome d'ansia generalizzata & & & & & & \\
\hline (F41.1) & 2 & 2.0 & 3 & 1.3 & 16 & 6.2 \\
\hline \multicolumn{7}{|l|}{ Sindrome da somatizzazione } \\
\hline$(\mathbf{F} 45.0)$ & 0 & 0.0 & 1 & 0.3 & 0 & 0.0 \\
\hline Sindrome ipocondriaca (F45.2) & 0 & 0.0 & 1 & 0.8 & 0 & 0.0 \\
\hline Nevrastenia $(\mathrm{F} 48.0)$ & 1 & 1.0 & 9 & 3.0 & 6 & 1.6 \\
\hline Un disturbo psichico & 4 & 3.9 & 27 & 14.0 & 30 & 13.4 \\
\hline Due o più disturbi psichici & 0 & 0.0 & 15 & 4.8 & 13 & 5.5 \\
\hline Totale & 28 & 100.0 & 104 & 100.0 & 118 & 100.0 \\
\hline
\end{tabular}

* Stime ponderate 
campo occupazionale e nelle attività quotidiane. Contrariamente all'opinione tuttora diffusa, i disturbi psichici lamentati dai pazienti che si rivolgono ai servizi di medicina generale costituiscono un importante problema di salute pubblica e per essi si rendono necessari appropriati programmi d'intervento.

Ringraziamenti. Desideriamo ringraziare i medici di medicina generale che hanno gentilmente accettato di partecipare allo studio: Dr. Stefano Baccaglini, Dr. Claudio Bertini, Dr. Fausto Bodini, Dr. Mario Casella, Dr. Paolo Ferrari, Dr. Silvio Fratton, Dr. Massimo Gastaldo, Dr. Roberto Gianfreda, Dr. Marco Landoni, Dr. Guerino Locatelli, Dr. Gilberto Mantelli, Dr. Cipriano Mirandola, Dr. Ermanno Motta, Dr. Roberto Pecci, Dr. Silvano Pomari, Dr. Giuseppe Ricci. Siamo inoltre grati al Dr. Maurizio Bagnani e al Dr. Silvano Pedron, che hanno preso parte ad una fase preliminare dello studio.

I nostri più sentiti ringraziamenti vanno ai membri dell'Istituto di Psichiatria dell'Università di Verona per il loro prezioso contributo durante la raccolta dei dati: Dr. Francesco Amaddeo, Dr. Marco Bortolomasi, Sig.ra Fiorenza Bresaola, Dott.ssa Laura $\mathrm{Cu}$ nico, Sig.ra Marina Masetti, Dr. Stylianos Nicolaou, Dott.ssa Asunçion Sacristan Cascajo Rodriguez, Dott.ssa Anna Saltini, Dott.ssa Licia Scantamburlo.

Siamo infine grati ai due anonimi referees per la scrupolosa opera di revisione cui hanno sottoposto una versione precedente di questo lavoro e per gli utili consigli e suggerimenti forniti.

Questo lavoro è stato condotto grazie al finanziamento della Regione Veneto, Ricerca Sanitaria Finalizzata, Contratto $N^{\circ} 401-$ 01.93, concesso al Professor Michele Tansella.

\section{BIBLIOGRAFIA}

American Psychiatric Association (1980). Diagnostic and Statistical Manual of Mental Disorders (3rd ed.). American Psychiatric Association: Washington DC.

American Psychiatric Association (1987). Diagnostic and Statistical Manual of Mental Disorders (3rd ed. revised). American Psychiatric Association: Washington DC.

Babor T.F., de la Fuente J.R., Saunders J. \& Grant M. (1989). Audit: the Alcohol Use Disorders Identification Test. Guidelines for Use in Primary Health Care. World Health Organization: Geneva.

Balestrieri M., Meneghelli G. \& Tansella M. (1992). Assistenza psichiatrica e monitoraggio dei servizi. Il Registro dei casi di Verona-Sud 1987-1990. Epidemiologia e Psichiatria Sociale 1, 117-132.

Barsky A.J., Geringer E. \& Wool C.A. (1988). A cognitive-educational treatment for hypochondriasis. General Hospital Psychiatry 10, 322-327.

Berti Ceroni G., Berti Ceroni F., Bivi R., Corsino M.A., De Marco P., Gallo E., Giovannini G., Gherardi S., Pezzoli A., Rucci P. \& Neri C. (1992). DSM-III mental disorders in general medical sector: a follow-up and incidence study over a twoyear period. Social Psychiatry and Psychiatric Epidemiology 27, 234-241.

Bridges K. \& Goldberg D. (1985). Somatic presentation of DSMIII psychiatric disorders in primary care. Journal of Psychosomatic Research 29, 563-569.
Bridges K., Goldberg D., Evans B. \& Sharpe T. (1991). Determinants of somatization in primary care. Psychological Medicine 21, 473-483.

Cormier L.E., Katon W., Russo J., Hollifield M., Hall M.L. \& Vitaliano P.P. (1988). Chest pain with negative cardiac diagnostic studies: relationship to psychiatric illness. Journal of Nervous and Mental Disease 176, 351-358.

Croft-Jeffreys C. \& Wilkinson G. (1989). Estimated costs of neurotic disorder in UK general practice 1985. Editorial. Psychological Medicine 19, 549-558.

Escobar J.I., Burnam M.A., Karno M., Forsythe A. \& Golding J.M. (1987). Somatization in the community. Archives of General Psychiatry 44, 713-718.

Finlay-Jones R. \& Burvill P.W. (1978). Contrasting demographic patterns of minor psychiatric morbidity in general practice and the community. Psychological Medicine 8, 455-466.

Finlay-Jones R., Brown G.W., Duncan-Jones P., Haris J., Murphy E. \& Prudo R. (1980). Depression and anxiety in the community: replicating the diagnosis of a case. Psychological Medicine 10, 445-454.

Folstein M.F., Folstein F.E. \& McHugh P.R. (1975). Mini-Mental State: a practical method for grading the cognitive state of patients for the clinician. Journal of Psychiatric Research 12, 189-198.

Fylkesnes K. \& Forde O.H. (1991). The Troms6-study: predictors of self-evaluated health - Has society adopted the expanded health concept? Social Science and Medicine 32, 141-146.

Fylkesnes K. \& Forde O.H. (1992). Determinants and dimensions involved in self-evaluation of health. Social Science and Medicine 35, 271-279.

Gask L. (1992). Training general practitioners to detect and manage emotional disorders. International Review of Psychiatry 4, 293-300.

Gater R., de Almeida e Sousa B., Barrientos G., Caraveo J., Chandrashekar C.R., Dhadphale M., Goldberg D., Al Kathiri A.H., Mubbashar M., Silhan K., Thong D., Torres-Gonzales F. \& Sartorius N. (1991). The pathways to psychiatric care: a cross-cultural study. Psychological Medicine 21, 761-774.

Goldberg D.P. (1972). The Detection of Psychiatric Illness by Questionnaire. Maudsley Monograph $\mathrm{N}^{\circ} 21$. Oxford University Press: London.

Goldberg D.P. \& Bridges K. (1988). Somatic presentation of psychiatric illness in primary care setting. Journal of Psychosomatic Research 32, 137-144.

Goldberg D. \& Huxley P. (1992). Common Mental Disorders. A Bio-social Model. Tavistock/Routledge: London.

Goldberg D.P. \& Williams P. (1988). The User's Guide to the General Health Questionnaire. NFER/NELSON: Windsor, Slough.

Huxley P. \& Goldberg D. (1975). Social versus clinical prediction in minor psychiatric disorder. Psychological Medicine 5, 96100.

Johnson J., Weissman M.M. \& Klerman G.L. (1992). Service utilization and social morbidity associated with depressive symptoms in the community. Journal of the American Medical Association $267,1478-1483$.

Katon W., Ries R.K. \& Kleinman A. (1984). Part II: a prospective DSM-III study of 100 consecutive somatization patients. Comprehensive Psychiatry 25, 305-314.

Katon W., Lin E., Von Korff M., Russo J., Lipscomb P. \& Bush T. (1991). Somatization: a spectrum of severity. American Journal of Psychiatry 148, 34-40.

Klerman G.L. (1989). Depressive disorders: further evidence for increased medical morbidity and impairment of social functio- 
ning. Archives of General Psychiatry 46, 856-858.

Kruesi M.J.P., Dale J. \& Straus S.E. (1989). Psychiatric diagnoses in patients who have chronic fatigue syndrome. Journal of Clinical Psychiatry 50, 53-56.

Marino S., Bellantuono C. \& Tansella M. (1990). Psychiatric morbidity in general practice in Italy. A point-prevalence survey in a defined geographical area. Social Psychiatry and Psychiatric Epidemiology 25, 67-72.

Narrow W.E., Regier D.A., Rae D.S., Manderscheid R.W. \& Locke B.Z. (1993). Use of services by persons with mental and addictive disorders. Findings from the National Institute of Mental Health Epidemiologic Catchment Area Program. Archives of General Psychiatry 50, 95-107.

Ormel J., Oldehinkel T., Brilman E. \& Van Den Brink W. (1993). Outcome of depression and anxiety in primary care. A three -wave $3 \frac{1}{2}$-year study of psychopathology and disability. Archives of General Psychiatry 50, 759-766.

Piccinelli M. (1994). Disturbi psichici nella medicina generale: perché tante differenze? Epidemiologia e Psichiatria Sociale 3, 195-208.

Piccinelli M., Bisoffi G., Bon M.G., Cunico L. \& Tansella M. (1993). Validity and test-retest reliability of the Italian version of the 12-item General Health Questionnaire in general practice: a comparison between three scoring methods. Comprehensive Psychiatry 34, 198-205.

Regier D.A., Narrow W.E., Rae D.S., Manderscheid R.W., Locke B.Z. \& Goodwin F.K. (1993). The de facto US mental and addictive disorders service system. Epidemiologic Catchment Area prospective 1-year prevalence rates of disorders and services. Archives of General Psychiatry 50, 85-94.

Robins L.N. \& Regier D.A. (ed.) (1991). Psychiatric Disorders in America. The Epidemiologic Catchment Area Study. The Free Press: New York.

Robins L.N., Wing J.K., Wittchen H.U., Helzer J.E., Babor T.F., Burke J. \& Farmer A. (1988). The Composite International Diagnostic Interview. An epidemiologic instrument. $\boldsymbol{A r}$ chives of General Psychiatry 45, 1069-1077.

Rosenblatt R.A., Cherkin D.C., Schneeweiss R. \& Hurt L.G. (1983). The content of ambulatory medical care in the United States. An interspecialty comparison. New England Journal of Medicine 309, 892-897.

Roy R., Thomas M. \& Matas M. (1984). Chronic pain and depression: a review. Comprehensive Psychiatry 25, 96-105.

Sartorius N. \& Üstün T.B. (in press). Mental Illness in Primary Care. An International Study. John Wiley \& Sons: New York.

Sartorius N., Davidian H., Ernberg G., Fenton F.R., Fujii I., Gastpar M., Gulbinat W., Jablensky A., Kielholz P., Lehman H.E., Naraghi M., Shimizu M., Shinfuku N. \& Takahashi R. (ed.) (1983). Depressive Disorders in Different Cultures. World Health Organization: Geneva.

Sartorius N., Jablensky A., Korten A., Ernberg G., Anker M., Cooper J.E. \& Day R. (1986). Early manifestations and firstcontact incidence of schizophrenia in different cultures. A preliminary report on the initial evaluation phase of the WHO collaborative study on determinants of outcome of severe mental disorders. Psychological Medicine 16, 909-928.

Sartorius N., Üstün T.B., Costa e Silva J.-A., Goldberg D., Lecrubier Y., Ormel J., Von Korff M. \& Wittchen H.-U. (1993). An international study of psychological problems in primary care. Archives of General Psychiatry 50, 819-824.

Sharp D. \& Morrel D. (1989). The psichiatry of general practice. In The Scope of Epidemiological Psychiatry (ed. P. Williams, G. Wilkinson and K. Rawnsley), pp. 404-419. Routledge: London.
Shepherd M. \& Clare A. (1981). Psychiatric Illness in General Practice, 2nd ed. Oxford University Press: Oxford.

Shepherd M., Cooper B., Brown A.C. \& Graham K. (1966). Psychiatric Illness in General Practice. Oxford University Press: Oxford.

Simon G.E. \& Von Korff M. (1991). Somatization and psychiatric disorder in the NIMH Epidemiologic Catchment Area Study. American Journal of Psychiatry 148, 1494-1500.

Smith G.R., Monson R.A. \& Ray D.C. (1986). Psychiatric consultation in somatization disorder. A randomized controlled study. New England Journal of Medicine 314, 1407-1413.

Stewart A.L., Hays R.D. \& Ware J.E. (1988). The MOS short form general health survey: reliability and validity in a patient population. Medical Care 26, 724-735.

Sullivan M.D., Katon W., Dobie R., Sakai C., Russo J. \& Harrop-Griffiths J. (1988). Disabling tinnitus: association with affective disorder. General Hospital Psychiatry 10, 285-291.

Tacchini G., Coppola M.T., Musazzi A., Altamura A.C. \& Invernizzi G. (1994). Validazione multinazionale della Composite International Diagnostic Interview (CIDI). Minerva Psichiatrica 35, 63-80.

Tansella M. \& Williams P. (1989). The spectrum of psychiatric morbidity in a defined geographical area. Psychological Medicine $19,765-770$.

Van Hemert A.M., Hengeveld M.W., Bolk J.H., Rooijmans H.G.M. \& Vandenbroucke J.P. (1993). Psychiatric disorders in relation to medical illness among patients of a general medical out-patient clinic. Psychological Medicine 23, 167-173.

Vazquez-Barquero J.L., Wilkinson G., Williams P., Diez-Manrique J.F. \& Pena C. (1990). Mental health and medical consultation in primary care settings. Psychological Medicine 20, 681-694.

Walker E., Katon W., Arrop-Griffiths J., Holm L., Russo J. \& Hickock L.R. (1988). Relationship of chronic pelvic pain to psychiatric diagnoses and childhood sexual abuse. American Journal of Psychiatry 145, 75-80.

Ware J.E.J. \& Sherbourne C.D. (1992). The MOS 36-item short form health survey (SF-36). I. Conceptual framework and item selection. Medical Care 30, 473-483.

Warwick H.M.C. (1989). A cognitive-behavioral approach to hypochondriasis and health anxiety. Journal of Psychosomatic Research 33, 705-711.

Wells K.B., Golding J. \& Burnam M.A. (1988). Psychiatric disorder and limitations in physical functioning in a sample of the Los Angeles general population. American Journal of Psychiatry 145, 712-717.

Wells K.B., Stewart A., Hays R.D., Burnam M.A., Rogers W., Daniels M., Berry S., Greenfield S.\& Ware J. (1989). The functioning and well-being of depressed patients. Results from the Medical Outcome Study. Journal of the American Medical Association 262, 914-919.

Wells K.B., Rogers W., Burnam M.A., Greenfield S. \& Ware Jr. J.E. (1991). How the medical comorbidity of depressed patients differs across health care settings: results from the Medical Outcome Study. American Journal of Psychiatry 148, 1688-1696.

Wiersma D., DeJong A. \& Ormel J. (1988). The Groningen Social Disability Schedule: development, relationship with ICIDH and psychometric properties. International Journal of Rehabilitation Research 3, 213-224.

Wilkinson G., Smeeton N., Skuse D. \& Fry J. (1988). Consultation for physical illness by patients diagnosed and treated for psychiatric disorders by a general practitioner: a 20 year follow up study. British Medical Journal 297, 776-778. 
Williams P., Tarnopolsky A., Hand D. \& Shepherd M. (1986). Minor Psychiatric Morbidity and General Practice Consultation: The West London Survey. Psychological Medicine Monograph Supplement No. 9. Cambridge University Press: Cambridge.

Wing J.K. \& Sturt E. (1978). The PSE - ID - CATEGO System Supplementary Manual. MRC Social Psychiatry Unit, Institute of Psychiatry: London.

Wohlfarth T.D., Van den Brink W., Ormel J., Koeter M.W. \& Oldehinkel A.L. (1993). The relationship between social dysfunctioning and psychopathology among primary care attenders. British Journal of Psychiatry 163, 37-44.

World Health Organization (1978). Primary Health Care, Report of the International Conference on Primary Health Care in
Alma Ata, 1978. Health for All Series, $\mathrm{N}^{\circ} 1$. World Health Organization: Geneva.

World Health Organization (1980). WHO Psychiatric Disability Assessment Schedule (WHO/DAS). World Health Organization: Geneva.

World Health Organization (1990a). The Introduction of a Mental Health Component into Primary Health Care. World Health Organization: Geneva.

World Health Organization (1990b). CIDI-Core. Composite International Diagnostic Interview, Core Version. World Health Organization: Geneva.

World Health Organization (1992). International Classification of Diseases, 10th Revision. World Health Organization: Geneva. 